\title{
Classical Dimers on Penrose Tilings
}

\author{
Felix Flicker®, ${ }^{*}$ Steven H. Simon, ${ }^{\dagger}$ and S. A. Parameswaran ${ }^{\ddagger}$ \\ Rudolf Peierls Center for Theoretical Physics, Oxford OX1 3PU, United Kingdom
}

(Received 25 February 2019; revised manuscript received 3 October 2019; published 8 January 2020)

We study the classical dimer model on rhombic Penrose tilings, whose edges and vertices may be identified as those of a bipartite graph. We find that Penrose tilings do not admit perfect matchings (defectfree dimer coverings). Instead, their maximum matchings have a monomer density of $81-50 \varphi \approx 0.098$ in the thermodynamic limit, with $\varphi=(1+\sqrt{5}) / 2$ the golden ratio. Maximum matchings divide the tiling into a fractal of nested closed regions bounded by loops that cannot be crossed by monomers. These loops connect second-nearest-neighbor even-valence vertices, each of which lies on such a loop. Assigning a charge to each monomer with a sign fixed by its bipartite sublattice, we find that each bounded region has an excess of one charge, and a corresponding set of monomers, with adjacent regions having opposite net charge. The infinite tiling is charge neutral. We devise a simple algorithm for generating maximum matchings and demonstrate that maximum matchings form a connected manifold under local monomerdimer rearrangements. We show that dart-kite Penrose tilings feature an imbalance of charge between bipartite sublattices, leading to a minimum monomer density of $(7-4 \varphi) / 5 \approx 0.106$ all of one charge.

DOI: 10.1103/PhysRevX.10.011005

\section{INTRODUCTION}

Dimer models are convenient abstractions of the physics of energetic constraints arising from strong correlations. Their solvability admits mathematically precise statements, which can then lend insight into a wide range of physical situations. The quantum dimer model was introduced as an approximate treatment of fluctuating nearest-neighbor spin singlets in the resonating-valence-bond state [1-4] proposed as a possible explanation for high-temperature superconductivity [5,6]. Describing spin configurations in terms of singlets naturally implies a hard-core constraint: A single site with a spin- $1 / 2$ degree of freedom can belong to at most one singlet defining a dimer model. Although the spin-dimer mapping is not one to one, the dimer model is nevertheless a useful caricature of the underlying spins, and an intuitive understanding in terms of dimers often translates fruitfully into spin models despite their more complicated structure.

\footnotetext{
*flicker@physics.org

†steven.simon@physics.ox.ac.uk

¥sid.parameswaran@physics.ox.ac.uk
}

Published by the American Physical Society under the terms of the Creative Commons Attribution 4.0 International license. Further distribution of this work must maintain attribution to the author(s) and the published article's title, journal citation, and DOI.
Subject Areas: Condensed Matter Physics,

Statistical Physics,

Strongly Correlated Materials
Work on dimer models also underpins research on topologically ordered states of matter [3,4]. A defining characteristic of such phases is fractionalization, a phenomenon whereby the emergent excitations of a system appear as fractions of the microscopic degrees of freedom [7]. In addition to its fundamental significance, fractionalization is also relevant to applications in which fractionalized quasiparticles can be used to perform quantum computation in a topologically protected manner [8]. Dimer models provide a particularly elegant framework within which to study such phenomena [9]. Monomersobtained by breaking apart dimers, and hence, fractionalized in an intuitive sense-can be thought of as sources and sinks of an emergent gauge field. Quantum fluctuations (resonances) between dimer configurations give the gauge field dynamics. Depending on the lattice structure and dimensionality, at long wavelengths the gauge field dynamics can either exhibit confinement or else can be described by a discrete or continuous gauge structure, respectively, characterized by gapped or gapless excitations $[3,4,10]$. In both cases, monomers may be separated to arbitrary distances at finite energy cost (they are deconfined): The system thus hosts emergent fractionalized quasiparticles.

The understanding of quantum dimer models frequently draws on highly influential exact results of their classical counterparts [11-15]. Insights are also afforded by numerical simulations of classical dimers $[16,17]$ that are often more computationally tractable than their quantum 
generalizations. Studying dimer coverings of graphs remains an active area of current research in mathematics and statistical physics [18-23]. For both the classical and quantum cases, results to date have focused primarily on periodic graphs, partly because of their relative simplicity and the resulting potential for exact results and partly because of the relevance to physical systems such as crystal lattices $[11,19]$. There is also active interest in investigating dimers on random graphs, such as those with quenched disorder [24-27].

Traditionally, these two extremes, periodicity and disorder, were the only cases studied in materials physics. This changed with the discovery of quasicrystals, states of matter with properties intermediate between the periodic order of crystals and the disorder of glasses. The identification of quasicrystals via their diffraction patternswhich feature discrete rotational symmetries forbidden in periodic crystals-led to a redefinition of crystallography in the second half of the 20th century [28]. Perhaps the simplest route to understanding quasicrystals is through aperiodic tilings such as the Penrose tiling (Fig. 1), which lacks the discrete translational invariance of periodic lattices featuring instead a discrete scale invariance [29-32]. Quasicrystals are real materials with the symmetries of Penrose-like tilings, just as crystals are real materials with the space-group symmetries of periodic lattices [33]. Although a large body of work has explored single-particle phenomena in quasiperiodic systems [34-36], including more recent extensions to incorporate topological properties [37-39], few studies have explored strongly correlated phenomena in quasicrystals. Recent interest in understanding many-body localization in quasiperiodic systems [40-42], as well as the relevance of quasicrystals to magnetic insulators, heavy fermion materials [43-47],

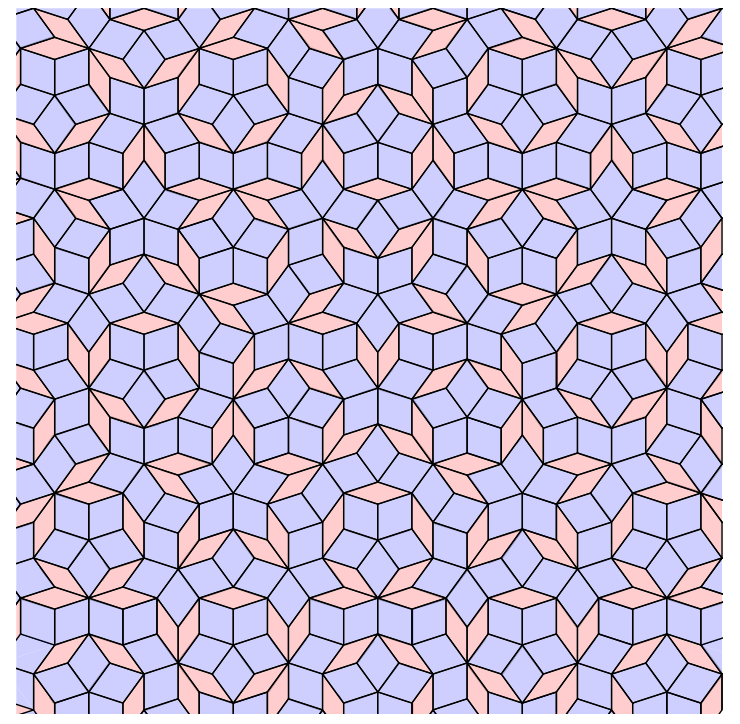

FIG. 1. A finite section of the Penrose tiling constructed of two rhombuses (colored red and blue here). and even superconductivity [48], suggest that the time is ripe to investigate such problems.

Here, we combine these two distinct lines of investigation and extend the study of dimer models to include quasiperiodic graphs. The reason for studying dimer models is twofold: On the one hand, they account for the physics of magnetic frustration and local constraints (textbook correlation effects) from the outset, and, on the other hand, our analysis can leverage insights from combinatorial graph theory. Specifically, we consider classical dimers on Penrose tilings, with the vertices and edges of the tiling considered the vertices and edges of a graph. Perhaps unsurprisingly, this case proves fundamentally distinct from both periodic and disordered graphs. We prove a number of exact results. For the majority of the paper, we consider the Penrose tiling constructed from two rhombic tiles shown in Fig. 1. For this system, we prove that it is not possible to achieve a perfect matching of dimers to vertices, such that each vertex touches precisely one dimer. We then turn to maximum matchings, in which the maximum number of dimers appears in the graph, with no vertex connected to two dimers. We prove that the density of monomer defects, vertices not reached by dimers, is $81-50 \varphi$, with $\varphi=(1+\sqrt{5}) / 2$ the golden ratio. We provide an algorithm for generating maximum matchings. Considering the monomers as mobile particles with motion defined by a local reconfiguration of dimers, we prove that monomers are restricted to closed finite regions of the graph, which appear in a nested fractal structure. We prove that maximum matchings form a manifold connected by local monomer-dimer moves. Turning briefly to the wider class of Penrose-like tilings, we prove that a variation on the Penrose tiling made instead from tiles shaped as darts and kites is also unable to admit perfect matchings. We prove that the minimum monomer density is precisely $(7-4 \varphi) / 5$ in this case and that all monomers are of the same bipartite charge. Considering aperiodic tilings other than Penrose, we provide evidence in support of our conjecture that certain examples admit perfect matchings. On the other hand, we prove that certain other examples cannot admit perfect matchings. We demonstrate that these latter cases feature broadly similar behavior to the rhombic Penrose tiling.

This paper proceeds as follows. In Sec. II, we provide background on Penrose tilings and dimer matchings of graphs. In Sec. III, we prove that Penrose tilings do not admit perfect matchings, i.e., they must feature a finite density of monomer defects, and study properties of the boundaries which restrict the movement of monomers. In Sec. IV, we provide an algorithm for generating maximum matchings. In Sec. $\mathrm{V}$, we find the minimum density of monomers in the infinite Penrose tiling analytically and numerically confirm the result. In Sec. VI, we demonstrate that maximum matchings form a manifold connected by local monomer-dimer moves. In Sec. VII, we consider 
classical dimers on other Penrose-like tilings. Finally, in Sec. VIII, we provide concluding remarks.

\section{BACKGROUND}

\section{A. Penrose tilings}

Penrose tilings are aperiodic covers of the Euclidean plane by sets of inequivalent tiles [29-31]. Throughout most of this paper, we take as the set two rhombuses (the so-called $P 3$ tiling shown in Fig. 1). Other Penrose tilings can be created as decorations of the $P 3$ tiling, as shown in Fig. 2(c); one such example, the $P 2$ tiling whose tiles are darts and kites, we consider in Sec. VII. Unless otherwise stated, "Penrose tiling" is assumed to mean the rhombic tiling. Penrose tilings lack the discrete translational invariance of periodic lattices, featuring instead a discrete scale invariance. They were originally devised as a problem in recreational mathematics, extending attempts to create aperiodic tilings begun by Kepler [49]. The study of Penrose tilings became relevant to physics with the observation that certain alloys demonstrate closely related symmetries [28]. The resulting quasicrystals can be identified by their diffraction patterns, which feature five-, eight-, ten-, or 12-fold rotational symmetries [50], in violation of the crystallographic restriction theorem which states that periodic structures in $2 \mathrm{D}$ or $3 \mathrm{D}$ can feature only two-, three-, four-, or sixfold rotations [51].

The Penrose tiling can be composed of the two rhombuses shown in Figs. 1 and 2. The two rhombuses have internal angles (as multiples of $2 \pi / 10)\{2,3\}$ (thick, blue in

(a)
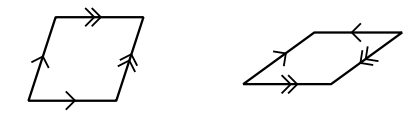

(b)

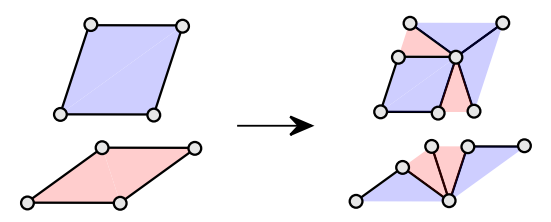

(c)

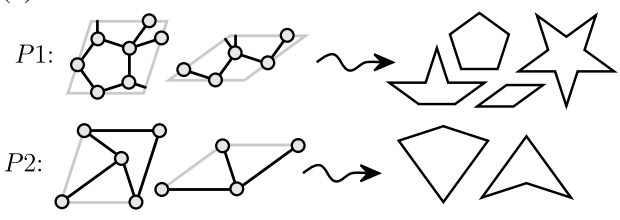

FIG. 2. (a) The Penrose tiling can be created by decorating the rhombuses with matching rules, where the decorations of neighboring edges must match. (b) An alternative method of creating the tiling uses inflation rules, in which each tile is subdivided into a combination of the two tiles as shown. Black lines indicate graph edges and gray circles graph vertices. (c) Decorating the tiles leads to different Penrose tilings made from different tile types: the $P 1$ pentagonal tiling and the $P 2$ dartkite tiling. the figures) and $\{1,4\}$ (thin, red in the figures). In order to force the tiling to be aperiodic, so-called matching rules must be applied to the tiles [Fig. 2(a)]: decorations of the edges such that only like edges may meet in the tiling [52]. Any tiling obeying the matching rules is a Penrose tiling; however, starting from a finite seed and growing the tiling by locally adding new tiles at the edge, it is possible to reach arrangements in which the tiling cannot be grown any further [30-32]. Figure 3 shows the eight possible ways in which the tiles can meet at a vertex, consistent with the matching rules.

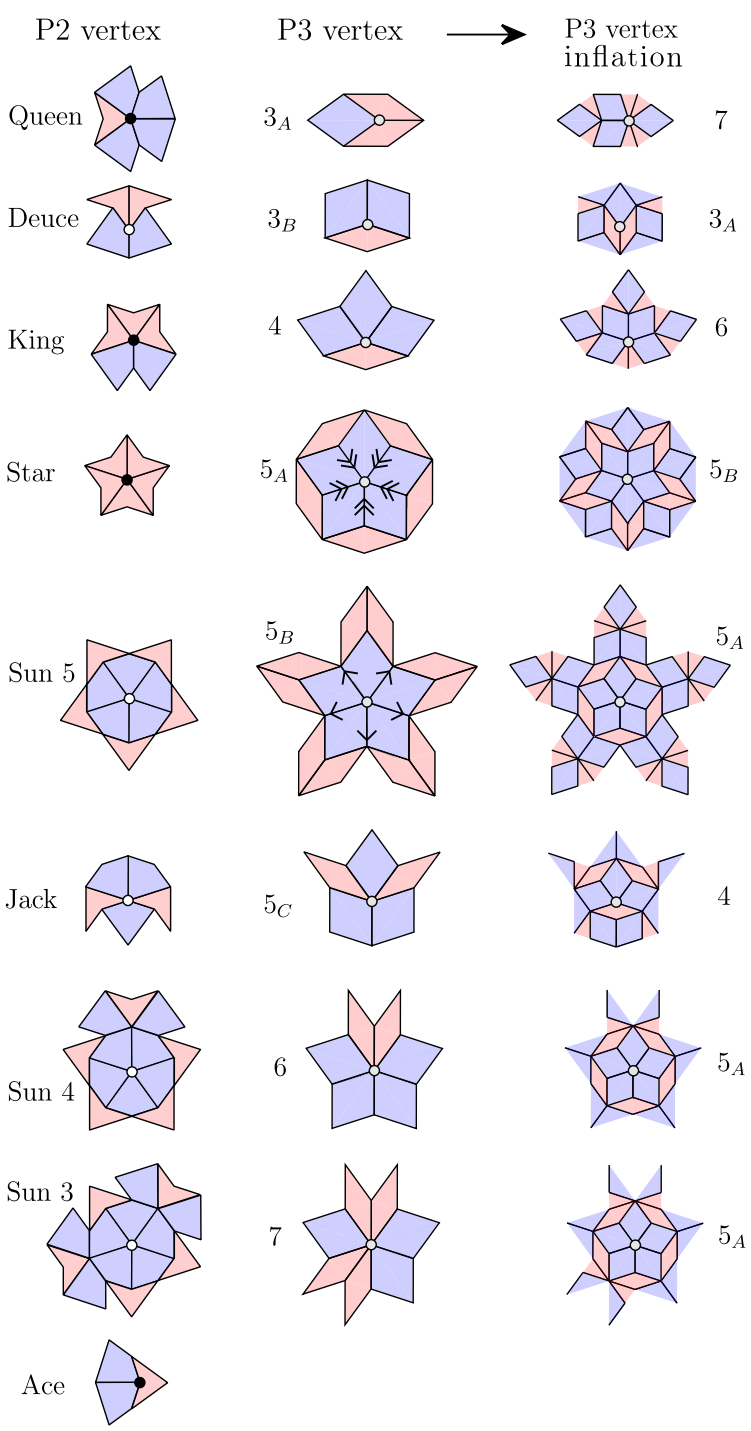

FIG. 3. The possible vertices in the $P 2$ dart-kite tiling (left), their equivalents in the $P 3$ rhombic tiling (middle), and the inflations of each (right). We label the $P 3$ vertices according to the number of edges connected to them. Tile matching rules distinguish the $5_{A, B}$ vertices and are indicated; $P 2$ suns are labeled according to the number of darts connected to them [53]. The $P 2$ vertices divide into two bipartite sublattices: star-queen-king-ace (black vertices) and deuce-jack-suns (white vertices). 
An alternative, recursive, algorithm for generating Penrose tilings is illustrated in Fig. 2(b). In this approach, rules are defined for subdividing each rhombus into a combination of the two rhombuses, in a process called inflation [32]. The resulting combination is then rescaled so as to be constructed of exact copies of the original rhombuses (the rescaling is not shown here). The inflations of the eight vertex types are shown in Fig. 3. An infinite number of inflations applied to any finite simply connected tile set constructed from the tiles in Fig. 2 results in a Penrose tiling. This construction also makes apparent the discrete scale invariance of the Penrose tiling: Inflation leads to a locally isomorphic tiling, meaning that every finite set of tiles found in one can be found in the other [54]. The presence of triangles (half-rhombuses) in the base inflation units leads to similar triangles on boundaries upon inflation, but these are negligible in the thermodynamic limit. Denoting the number of thick (blue) and thin (red) tiles after $n$ inflations as $b_{n}$ and $r_{n}$, respectively, their growth can be characterized by a $2 \times 2$ matrix:

$$
\left(\begin{array}{c}
b_{n+1} \\
r_{n+1}
\end{array}\right)=\left(\begin{array}{ll}
2 & 1 \\
1 & 1
\end{array}\right)\left(\begin{array}{l}
b_{n} \\
r_{n}
\end{array}\right) \text {. }
$$

Under repeated applications of the matrix, i.e., repeated inflations of the tiling, we find

$$
\left(\begin{array}{c}
b_{n} \\
r_{n}
\end{array}\right)=\left(\begin{array}{ll}
2 & 1 \\
1 & 1
\end{array}\right)^{n}\left(\begin{array}{l}
b \\
r
\end{array}\right)=\left(\begin{array}{l}
F_{2 n+1} b+F_{2 n} r \\
F_{2 n} b+F_{2 n-1} r
\end{array}\right) \text {, }
$$

where the initial numbers of tiles are $b$ and $r$. The growth of the number of tiles is controlled by the Fibonacci numbers $F_{n}($ for $n \geq 0)$ :

$$
\begin{aligned}
n & =0,1,2,3,4,5,6,7,8,9,10, \ldots, \\
F_{n} & =0,1,1,2,3,5,8,13,21,34,55, \ldots
\end{aligned}
$$

The largest eigenvalue of the matrix is $\varphi^{2}$, with $\varphi=(1+\sqrt{5}) / 2$ the golden ratio. $\varphi$ is a PisotVijayaraghavan (PV) number: a number with modulus greater than 1, whose Galois conjugates all have modulus strictly less than $1[30,55,56]$. Any integer power of a PV number is also a PV number. All quasicrystals and Penroselike tilings can be generated by inflation, and in all cases the largest eigenvalue of the inflation matrix is a quadratic irrational PV number $[57,58]$. In the thermodynamic limit, the largest eigenvalue dominates, and the ratio of the components of the associated right eigenvector gives the ratio of the number of tiles of each type. For the Penrose tiling, this ratio of thick to thin tiles is $\varphi$.

While the aperiodic nature of the tiling requires ambiguity in tile placements, the matching rules of Fig. 2(a) may force certain tile placements given certain local configurations. The set of tiles which unambiguously appears around a given feature is known as an empire [32]. In general, the empire of a feature will not be simply connected; the set of tiles simply connected to the feature is known as the local empire. Figure 4 shows the local empires of each vertex type in the Penrose tiling [59]. These play a major role in our discussion and crucially enable us to prove general results of the entire tiling.

\section{B. Dimer coverings}

There is extensive literature on dimer coverings of graphs in both physics and mathematics. Here we present only the points salient to the remaining discussion in this paper; for a general introduction to the topic, see Refs. $[14,19,60]$, and for an introduction to the relevance to physics, see Refs. [20,61].

A graph is a set of vertices connected by edges. We consider planar graphs, which can be embedded in the Euclidean plane such that no edges pass under or over one another. The graphs we consider are also bipartite, meaning that the vertices can be colored one of two colors, say, red and blue, such that the edges connect only red vertices to blue and vice versa (see Fig. 5). An equivalent statement is that all cycles (loops or closed paths) on the graph are of even length [60].

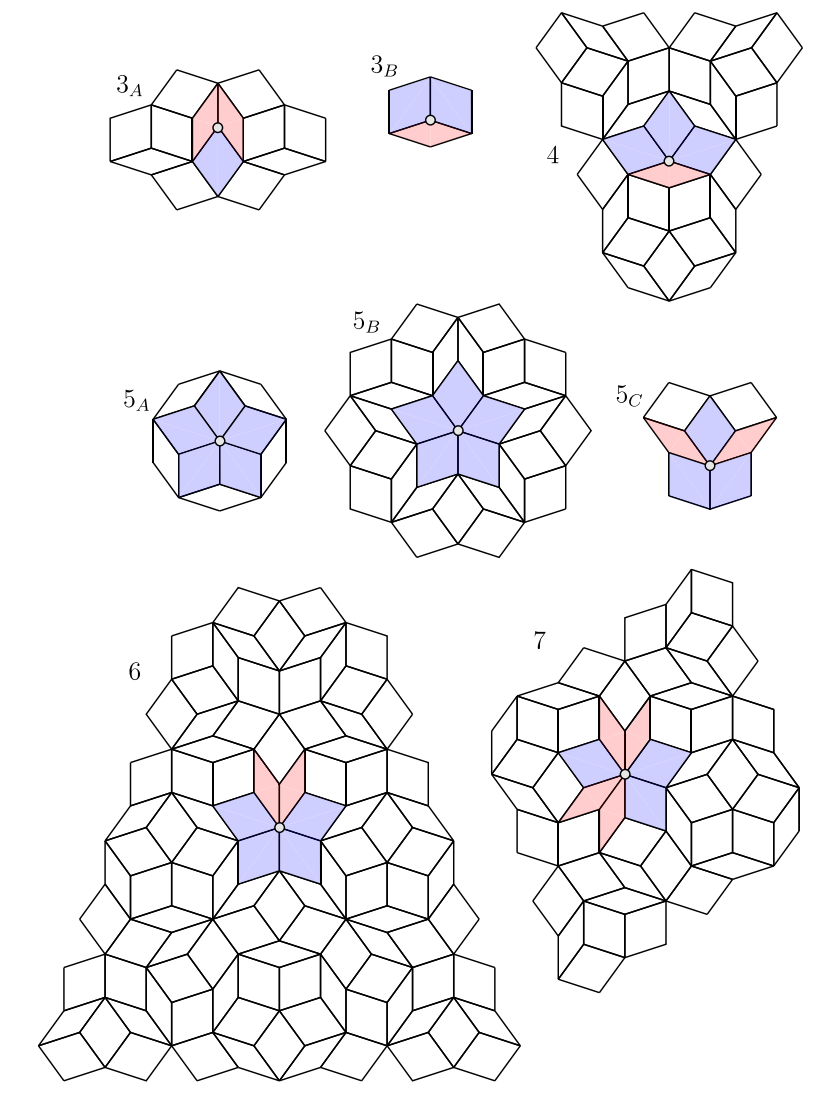

FIG. 4. Each vertex type in Fig. 3 is accompanied by a set of tiles which always appears around it, termed a local empire [32]. 
(a)

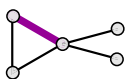

(b)

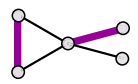

(c)

d)
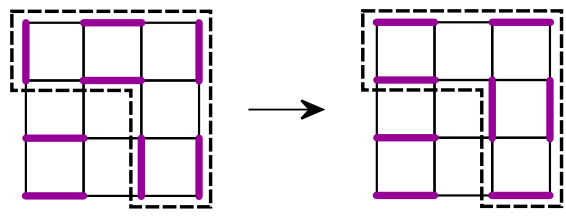

(e)
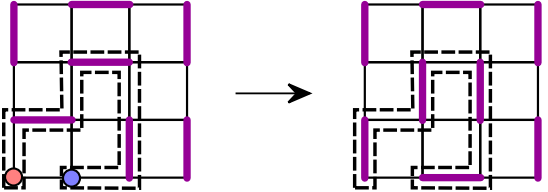

(f)
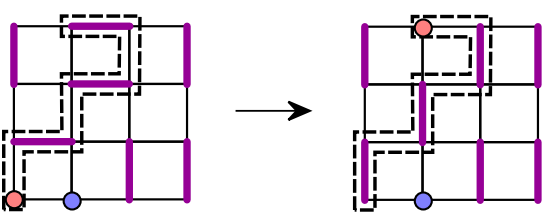

FIG. 5. (a) A maximal matching of a graph is a dimer covering such that no further edge can be covered with a dimer (purple) without causing a vertex (gray circles) to connect to two dimers. (b) A maximum matching additionally contains the maximum number of dimers. (c) If every vertex in a maximum matching connects to a dimer, the result is a perfect matching. (d) An alternating cycle is enclosed by the dashed line in this perfect matching of a bipartite graph. Augmenting the cycle (switching which edges are covered by dimers) results in another perfect matching. (e) Deleting one dimer results in a monomerantimonomer pair. Augmenting paths are alternating paths with both ends terminating on monomers. Augmenting the path removes both monomers. No augmenting path can be present in a maximum matching. (f) An alternating path terminating on one monomer is highlighted. Augmenting the path moves the monomer. We term a minimal monomer move the augmentation of an alternating path of minimum length, which enacts a monomer hop across one unoccupied and one occupied edge.

A matching of a graph is a set of edges such that no vertex touches more than one edge in the set [60]. A matching is equivalent to a hard-core dimer covering, and henceforth, we use the terms interchangeably and consider an edge to be covered by a dimer if it belongs to the matching. A maximal matching is a matching in which no further edges can be covered by dimers while remaining a matching [Fig. 5(a)]. We focus here on maximum matchings, maximal matchings with the additional property that the dimers cover the maximum possible number of edges [Fig. 5(b)]. A perfect matching is a maximum matching in which every vertex connects to a dimer [Fig. 5(c)]. There may be more than one perfect matching. Starting from a perfect matching of a bipartite graph (if one exists), deleting one dimer leads to two monomer defects where vertices connect to zero dimers [Figs. 5(d)-5(f)]. A maximum dimer matching hosts the minimum number of monomers [60]. Various algorithms exist for generating maximum matchings of graphs, such as the Hopcroft-Karp algorithm for bipartite graphs [62]. Along an alternating path, edges are alternately covered and not covered by dimers [63]. If the path closes, it is an alternating cycle. If it does not close, one end necessarily terminates on a monomer. An augmenting path is an alternating path with both ends terminating on monomers. In a bipartite graph, two monomers connected by an augmenting path must be of opposite bipartite charge; we term one a monomer and the other an antimonomer by analogy to particles. To augment a general alternating path is to switch which edges are covered by dimers and which are not. These cases are shown in Figs. 5(d)-5(f).

The creation of two monomers can be seen as analogous to the creation of a particle-antiparticle pair, with the charge of the particle being its bipartite color (red or blue). Rearranging dimers can have the effect of moving monomers; specifically, augmenting an alternating path terminating on a monomer moves the monomer along the length of the path. We define a minimal monomer move to be a hop across one unoccupied edge and one occupied edge, i.e., augmenting a shortest-length alternating path terminating on the monomer. As the monomer moves along the path, it switches which edges are covered by the dimers. An augmenting path connecting a monomer to an antimonomer can be thought of as a classical version of the Dirac strings connecting magnetic monopoles to their antimonopoles required by gauge consistency [64]. In a quantum gauge theory, these Dirac strings (which ensure the single valuedness of the wave function of an electron passing around the string) would be gauge-dependent quantities requiring a precise relationship between the electric and magnetic charge quanta in order to be unobservable. This ambiguity can survive in the graph setting in the following sense: Presented with a configuration of monomers and antimonomers, it may not be possible to make an unambiguous statement as to where the strings of flipped dimers lie. The dimers in a liquid phase can be seen as a structured vacuum in which monomers move.

In this paper, we identify the edges and vertices of the Penrose tiling with the edges and vertices of a graph and seek properties of dimer coverings of this graph. While we are unaware of previous work on this subject, some results on more general planar bipartite graphs can be applied to this case, as in Refs. [20,65,66]. Interacting spins on Penrose-like tilings were considered in Refs. [44,45,67-70]. The Hubbard model is treated on Penrose tilings in Ref. [71], building on earlier observations in both Penrose tilings [72] and other systems [73,74]. Intriguingly, Ref. [71] finds a finite density of localized electronic zero modes which exactly matches the density of monomers we identify here. This seemingly miraculous coincidence is in fact a result of a theorem due to Longuet-Higgins [75], which we explore in upcoming work [76]. Dimers on one-dimensional quasilattices are discussed in Ref. [77]. Treated as a graph in this way, the Penrose tiling of course admits a planar embedding, and we maintain the geometry of the tiling in the remaining 
discussion (although the results presented depend solely on the network topology of the graph). As the faces are all rhombuses, it follows that the graph is also bipartite: This is true for all planar graphs whose faces all have an even number of edges $[78,79]$.

\section{IMPOSSIBILITY OF PERFECT MATCHINGS ON PENROSE TILINGS}

Considering that the number of edges emanating from a vertex can range from three to seven in the Penrose tiling, it may not seem surprising that the corresponding graph does not admit a perfect matching. On the other hand, the graph is bipartite, and all cycles are of even length. The tiling can be constructed as a 2D slice through a 5D simple-hypercubic lattice; the graph equivalent of this higher-dimensional lattice would admit perfect matchings [30,31].

Finite sections of Penrose tilings can be created by inflating simply connected sets of tiles using the inflation rules in Fig. 2. Maximum dimer matchings can then be found, for example, using the Hopcroft-Karp algorithm. However, monomers resulting from this process may in principle be able to hop to the boundary by a sequence of minimal monomer moves (i.e., by augmenting alternating paths connecting each monomer to the boundary). In this case, it is unclear whether the monomers were an artifact of considering only a finite section of the tiling.

One statement about the Penrose tiling, however, follows straightforwardly from its bipartite structure: namely, that any matching on the rhombic Penrose tiling is charge neutral in the thermodynamic limit.

Proof.-First note that the trivial matching with zero dimers (monomers on all vertices) is charge neutral. This is because the average number of edges protruding from a vertex (the vertex valence) is the same for both bipartite sublattices. The average valence across the entire graph must be four, as all tiles have four edges. The average valence of the two bipartite sublattices must be equal because all vertex types appear in both sublattices (easily checked in a finite region). Then note that any matching is formed by placing dimers onto this trivial matching. Each dimer eliminates one monomer of each bipartite charge and therefore conserves total charge.

We now prove that a perfect matching of the Penrose tiling is not possible, as the structure of the tiling leads to closed loops of edges which can never be covered by dimers in maximum matchings. These loops cannot be crossed by alternating paths in maximum matchings and so act as impenetrable obstacles to monomer movement when the movement is defined via minimal monomer moves. The existence of a net imbalance of bipartite charge enclosed by such a loop then suffices to demonstrate the impossibility of perfect matchings. In the next subsection, we often simply state the key results that we rely on and (where appropriate) sketch the key ideas behind the proofs, relegating the details to the Appendix A.

\section{A. Impermeable monomer membranes}

We begin by observing that any even-valence vertex in the Penrose tiling (a 4-vertex or 6-vertex) has no even-valence neighbors and precisely two even-valence second-nearest neighbors (see Fig. 1) [80]. A proof of this statement relies on considering the local empire of the 6-vertex in Fig. 4, which is large enough to cover the entire tiling when allowing for overlaps [81].

A corollary of this result is that these chains of evenvalence vertices must either form closed loops or cross the entire system. We explain this fact and consider further properties of the loops in Sec. III B. The smallest such loop takes the form $(4-)^{5}$. We term this configuration a " 45 loop"; we later show that it is the only exception to various rules. Here we demonstrate that even-valence loops act as impermeable barriers to monomers within the set of maximum matchings. In order to do so, we first note that even-valence vertices never host monomers in maximum matchings.

Proof.-A full proof of this statement is provided in Appendix A. Here to set the stage of our discussion, we provide the flavor of the graphical arguments used in the proof (shown in Fig. 6) by considering the simplest

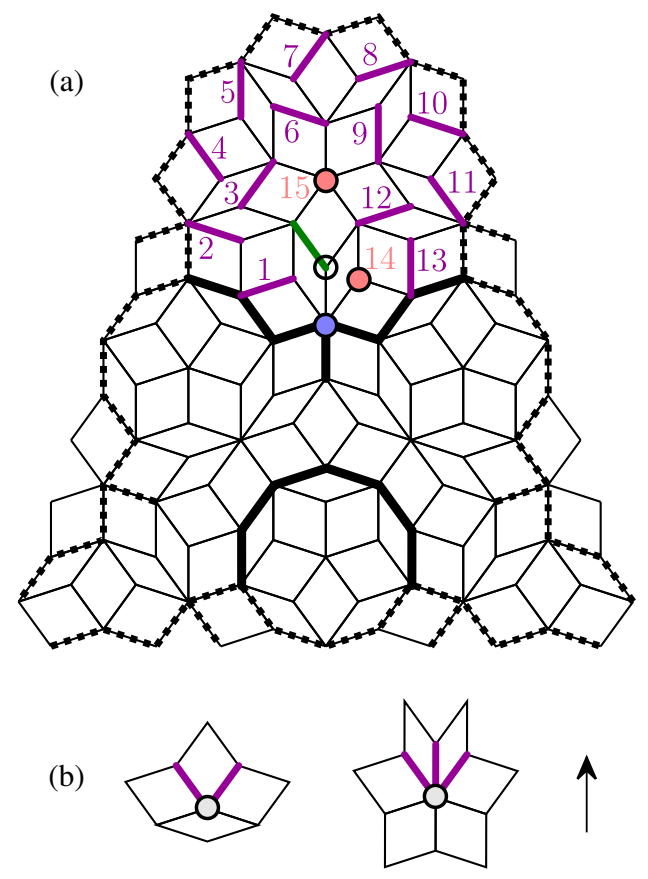

FIG. 6. Elements used in the proof that (a) a 6-vertex (blue) cannot host a monomer in a maximum matching, and (b) the dimer which must emanate from the 6-vertex must be placed on one of the three purple edges indicated. The direction of the 4- and 6-vertices is defined to align with the arrow. Thick black lines indicate edges which cannot be covered by dimers in maximum matchings. Solid (dashed) lines indicate definite (potential) uncoverable edges. Potential uncoverable edges are those that may or may not become uncoverable depending on how the local empire overlaps with others in the full tiling. 


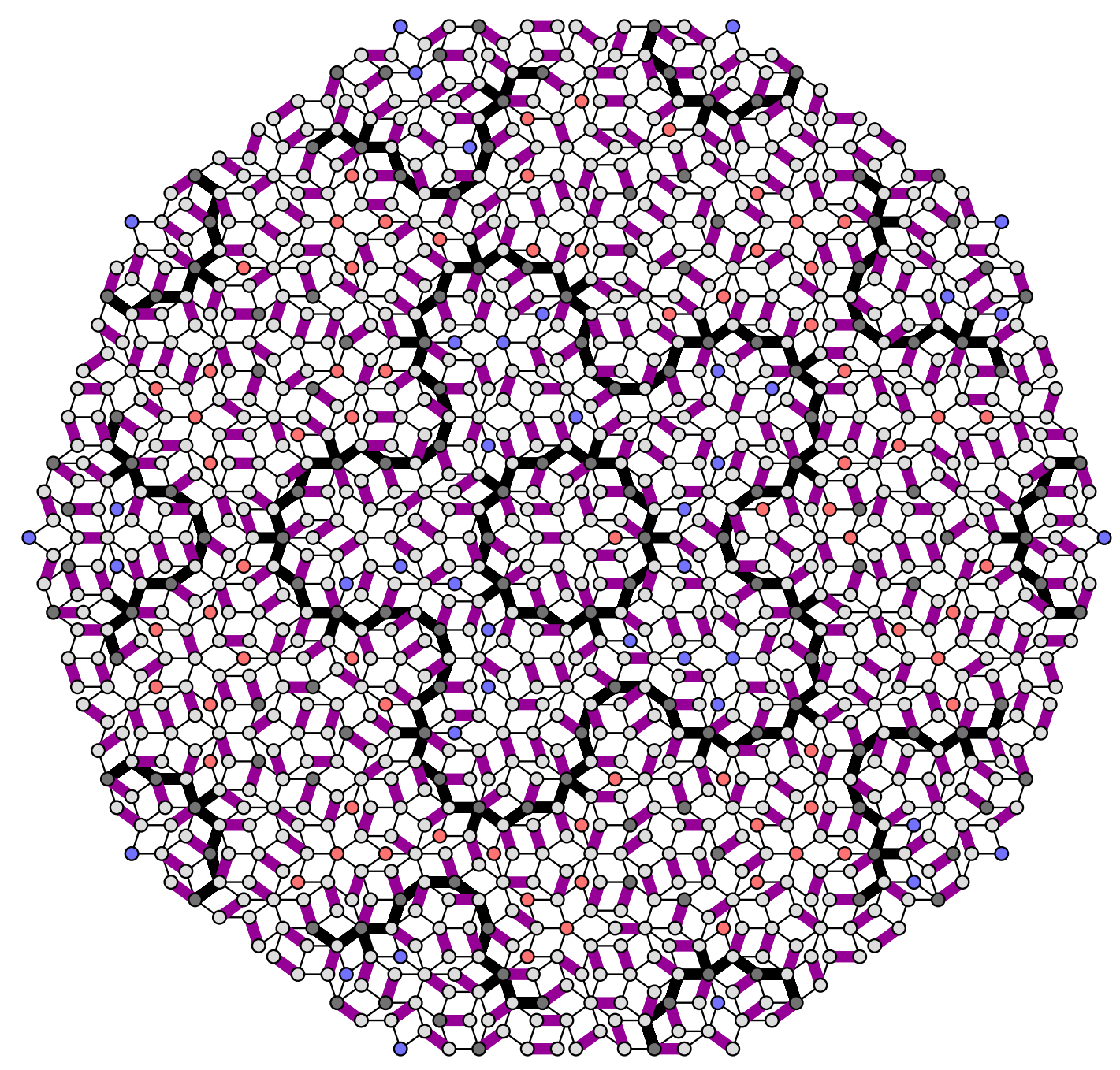

FIG. 7. A maximum matching of a finite section of the Penrose tiling. Certain edges (thick black lines) of even-valence vertices (dark gray) cannot be covered by dimers (purple) in maximum matchings. Monomers colored blue or red according to their bipartite charge cannot cross the closed thick black loops.

case, which is again that of the 6-vertex owing to the large size of its local empire. We begin by assuming that a monomer does exist on the 6-vertex [the blue vertex in Fig. 6(a)] and prove a contradiction: The monomer always connects via an augmenting path to an antimonomer. That is, placing the monomer on a 6-vertex implies a second monomer which is able to hop to the neighboring vertex via minimal monomer moves. Therefore, in a maximum matching the edge connecting these neighboring monomers will always be covered by a dimer. First, the presence of a monomer on the 6-vertex means that, by definition, no monomers can exist on neighboring vertices in a maximum matching. Consider the neighboring 3-vertex circled in black in Fig. 6(a): This must have a dimer connected to one of the two edges not connected to the 6-vertex. The two choices are equivalent under a vertical mirror symmetry. Cover the green edge with a dimer as shown. Proceeding clockwise, a chain of dimers (purple) is implied in the numerical order indicated. If any of these edges is not covered by a dimer, an antimonomer will result which connects via an augmenting path to the original monomer. At the end of this chain of implied dimers, the red monomer results. This monomer neighbors the original 6-vertex monomer and provides the desired contradiction, as the number of dimers in the matching can always be increased by covering the edge connecting the two monomers. No monomer can ever appear on a 6-vertex, and, by extension, on any vertex connected by an alternating path to a 6vertex, in a maximum matching. A similar argument (Appendix A 2, Fig. 19) demonstrates that 4-vertices also never host monomers in maximum matchings.

In proving the above result, we find a further restriction: namely, that in a maximum matching, monomers cannot be placed on the $5_{C}$-vertices which connect even-valence vertices, except in a $4^{5}$-loop, where at most one $5_{C}$-vertex can be covered by a monomer (a graphical proof is given in Appendix A 2, Fig. 20). We test these results numerically in finite systems by generating maximum matchings using the 


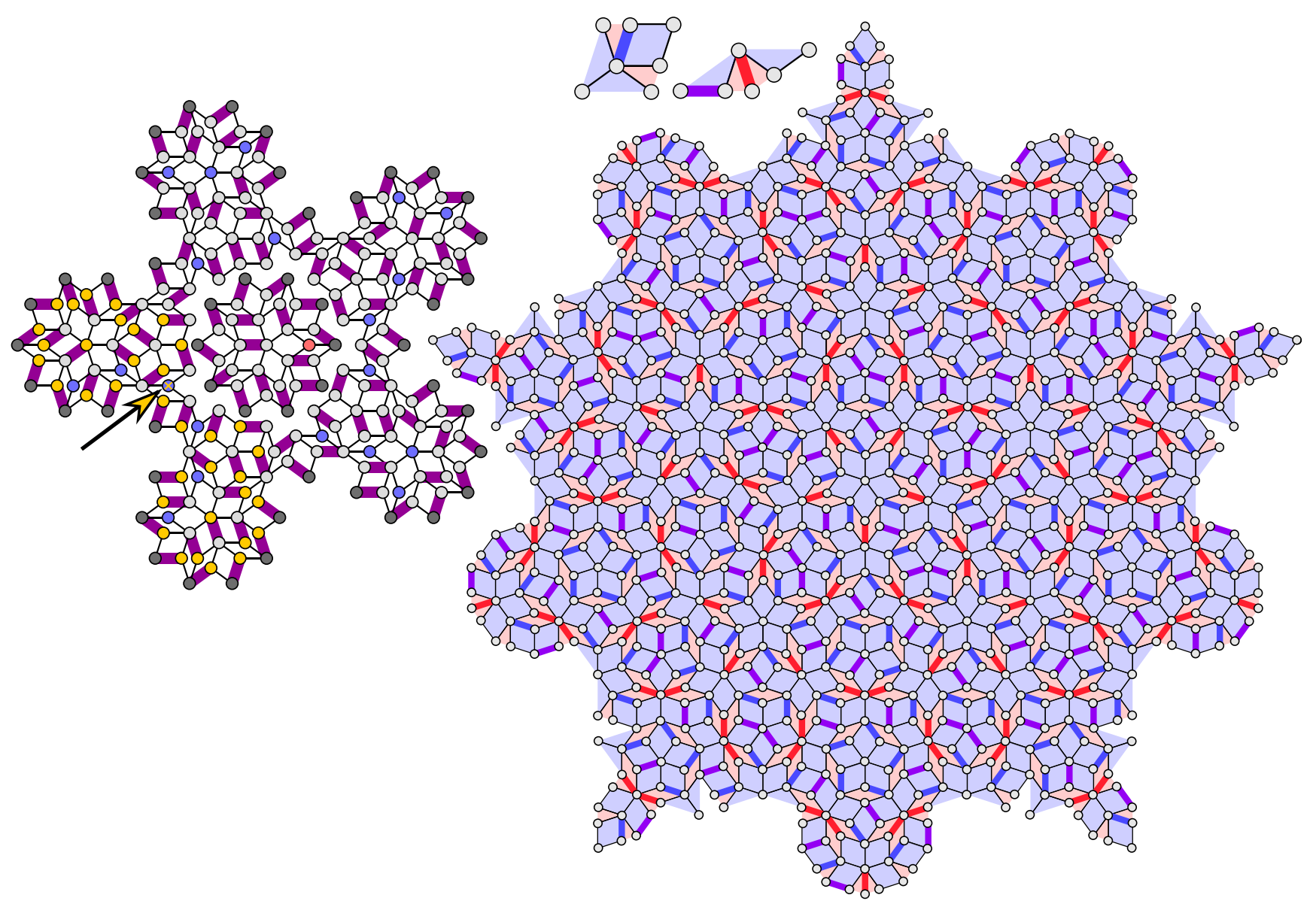

FIG. 8. (a) Deleting uncoverable edges in Fig. 7 disconnects the graph into monomer regions (the incomplete outermost region is removed). Neighboring monomer regions contain opposite net bipartite charge. The monomer indicated with a gold cross and arrow is able to reach the gold vertices by augmenting its alternating paths. It is not able to reach all vertices, even of its own charge; the obstruction is made by dimers rather than monomers (see Sec. VI). (b) Decorating the basic inflation elements with dimers in the final inflation results in the dimer covering shown. The following dimer inflation algorithm gives a maximum matching: Whenever a vertex is covered by two dimers, delete one. One monomer is then associated with each $5_{A, B}$-vertex with zero or one 7 -vertices as second-nearest neighbors, and three monomers are associated with each $5_{A}$-vertex with two 7 -vertices as second-nearest neighbors.

Hopcroft-Karp algorithm and verify that they satisfy these constraints.

The above arguments constrain the placement of dimers in maximum matchings: In any maximum matching, an even-valence vertex must always touch a dimer, which must always cover one of the edges indicated in Fig. 6(b). Figure 7 shows a finite section of Penrose tiling. Thick black lines indicate the edges which can never be covered by dimers in a maximum matching. As expected, these edges form closed loops connecting second-nearest-neighbor even-valence vertices but also include twigs protruding from the loops at the 6-vertices. The twigs capture the added constraints on dimer placement on 6-vertices shown in Fig. 6(b). We term these closed loops of second-nearestneighbor even-valence vertices connected via $5_{C}$-vertices monomer membranes. As we show in Appendix A 4, monomer membranes provide impermeable barriers to monomer motion. They play a central role in the remainder of our discussion.
The set of maximum matchings is unaffected if we delete the edges which can never be covered by dimers. As can be seen in Figs. 7 and 8, deleting these edges causes the graph to break into disconnected regions. Note that this illustrates the importance of the twigs - without deleting these, the different regions remain connected. A careful inspection reveals that there is an edge of the $5_{C}$-vertex, when the $5_{C}$-vertex appears in the configuration $-4-6-4-5_{C}-$ $4-4-6-4-$ (where only the relevant $5_{C}$-vertex is listed), which must also be deleted to cause the regions to disconnect. We prove in Appendix A 2 that the edge is indeed uncoverable by a dimer in maximum matchings precisely whenever it appears in this configuration. Each disconnected region is a subgraph containing an excess of one or the other bipartite charge. This fact forces a finite number of monomers of the corresponding excess charge in each region. Any two neighboring regions contain oppositely charged monomers. The edges of the $4^{5}$-loop can be covered by dimers, and so it does not result in disconnection. 
As we have mentioned, graphical proofs of the statements above are provided in Appendix A. They rely upon chains of dimers being implied by the matching rules subsequent to placing only two initial objects (a monomer and a dimer, or two dimers). These implication networks, as we term them, always exist, and always close: Once a dimer is implied, a whole chain must be implied, terminating only once the resulting network returns to the original dimer (we allow dimers to continue to be implied after a monomer has been implied). The proof follows from two observations: (i) Constraining two legs of a 3-vertex not to host dimers automatically implies a dimer on the third leg and (ii) at least two of the three vertices diametrically opposite a 3 -vertex (i.e., across the three rhombuses that meet at the vertex) are themselves 3-vertices. The monomer membranes contain no 3-vertices; they form the boundaries of the implication networks [82].

\section{B. Monomer membrane properties}

As monomer membranes are central to understanding the maximum matchings of the Penrose tiling, we provide further details of their properties here.

Figure 9 shows an alternative decoration of the two rhombuses with red and blue curves. Continuity of the curves enforces the tile matching rules (Fig. 2). Conway and Penrose independently demonstrated, that at most, two of the red curves may cross the entire tiling; all others form closed loops with $D_{5}$ symmetry ( $5 m$ in Hermann-Mauguin notation), which enclose regions of $D_{5}$ symmetry, each centered on either a $5_{A^{-}}$or $5_{B}$-vertex $[32,54]$. Every thick rhombus in the tiling is adjacent to precisely two other thick rhombuses, and therefore, chains of thick rhombuses also either cross the system or form closed curves. The red curves can be seen to follow the thick-rhombus chains. Monomer membranes also follow thick-rhombus chains, crossing the chains twice at each 6-vertex, and staying on the same side of the chains around 4-vertices. The proof again follows from the fact that the local empire of the 6-vertex shown in Fig. 9 can cover the entire tiling, allowing for overlaps. The results of Conway and Penrose therefore carry over to monomer membranes: At most two monomer membranes cross the entire system. All others are closed, with $D_{5}$ symmetry and bound $D_{5^{-}}$ symmetric sets of tiles. The proof that closed monomer membranes feature $D_{5}$ symmetry follows from the fact that each is generated by repeated inflation of a $5_{A^{-}}$or $5_{B^{-}}$ vertex, each of which has $D_{5}$ symmetry, and the fact that inflation about a $D_{5}$-symmetric point preserves the symmetry. As system-spanning loops cannot have $D_{5}$ symmetry, they cannot appear in any Penrose tilings created by inflating $D_{5}$-symmetric tile sets. As we focus primarily on such tile sets, the system-spanning loops will play a limited role in the subsequent discussion.

The local empire of the $5_{A}$-vertex is bounded by a $4^{5}$ loop, the smallest monomer membrane. All other monomer

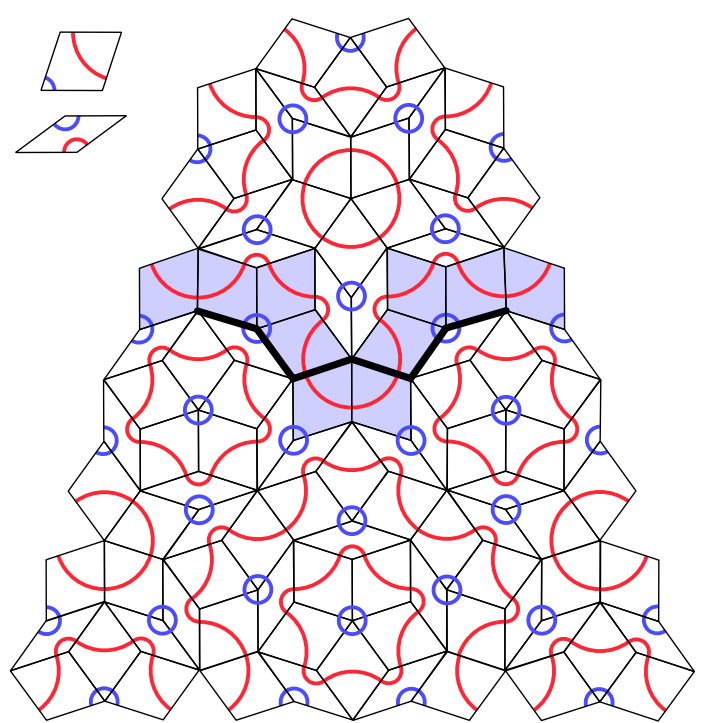

FIG. 9. Decorating the tiles with red and blue curves as indicated, continuity of the curves implies the matching rules of Fig. 2(a). At most, two red curves may cross the entire system [32,54]. All others must close around regions of $D_{5}$ symmetry. The curves follow chains of thick rhombuses, one of which is highlighted in blue (all thick rhombuses are adjacent to precisely two others). The curves also follow chains of second-nearestneighbor even-valence vertices (monomer membranes): a 4-6-4 segment is highlighted with a thick black line. Therefore, these chains also close around regions of $D_{5}$ symmetry centered on either a $5_{A}$ - or $5_{B}$-vertex. The thick black line can be seen to curve away from the 4 -vertex with curvature $2 \pi / 5$ and toward the 6 vertex with curvature $-4 \pi / 5$, with the directions of the vertices defined in Fig. 6.

membranes can be generated as inflations of this case [83]. Inflating the $4^{5}$-loop twice returns a (rotated) $4^{5}$-loop with some surrounding tiles. For subsequent inflations, the list of even-valence vertices in the outermost monomer membrane under inflation is

$$
4^{5} \rightarrow(46)^{5} \rightarrow(4644)^{5} \rightarrow(46444646)^{5} \rightarrow \ldots,
$$

where only the even-valence vertices in the loop are listed. The length of the boundary after $m$ inflations, as measured by the number of even-valence vertices it contains, is $5 \times 2^{m}$. This number is odd for $m=0\left(4^{5}\right.$, the only membrane permeable to monomers) and even for all $m>0$.

The specific sequences of 4's and 6's can be generated by the substitution rules:

$$
\begin{aligned}
& 4 \rightarrow 46, \\
& 6 \rightarrow 44 .
\end{aligned}
$$

If $n_{4,6}$ and $n_{4,6}^{\prime}$ denote the number of 4-, 6-vertices before and after inflation, we may assign a growth matrix as in Sec. II A: 


$$
\left(\begin{array}{l}
n_{4}^{\prime} \\
n_{6}^{\prime}
\end{array}\right)=\left(\begin{array}{ll}
1 & 2 \\
1 & 0
\end{array}\right)\left(\begin{array}{l}
n_{4} \\
n_{6}
\end{array}\right) .
$$

The largest eigenvalue of the growth matrix is 2 , confirming that the loop length doubles under inflation. Since the number is rational, the loops formed by an infinite number of inflations are not themselves quasilattices [84].

The total curvature of any loop must be $2 \pi$. The membranes curve away from the 4-vertex with curvature $2 \pi / 5$ (since the $4^{5}$-loop closes) and toward the 6 -vertex with curvature $-4 \pi / 5$ [since the $(46)^{5}$-loop also closes]. This fact can be seen, for example, in Fig. 9. Monomer membranes of increasing size come in two varieties: one centered on a $5_{A}$-vertex, and one centered on a $5_{B}$-vertex. The sequence of vertices appearing in each of these two varieties of membranes [Eq. (4)] can be generated by the following rule: Starting from a 4 (respectively, 46), generate the next term in the sequence by inserting (464) to the left of each symbol in the previous sequence. Considering just the minimal repeat unit (which appears five times in the full loop), gives

$$
\begin{aligned}
4 & \rightarrow(464) 4 \rightarrow(464) 4(464) 6(464) 4(464) 4 \rightarrow \ldots, \\
46 & \rightarrow(464) 4(464) 6 \rightarrow \ldots
\end{aligned}
$$

matching the sequence of Eq. (4), also generated by Eq. (5). Since (464) is curvature-free, this construction preserves the curvature of the seed. This guarantees a $\pm 2 \pi$ curvature for the loop. In principle, there are other sequences compatible with $D_{5}$ symmetry and closed loops, such as $(46464)^{5}$. This case proves incompatible with the tilematching rules of Fig. 2: Any $D_{5}$-symmetric region of a Penrose tiling must be centered on a $5_{A^{-}}$or $5_{B^{-}}$-vertex, but this sequence is centered on a decapod defect $[32,54,56]$.

The monomer membranes are fractal objects and are characterized by a fractal dimension $d_{F}$. We may define $d_{F}$ as follows: Upon rescaling the area $\mathcal{A}$ via

$$
\mathcal{A} \rightarrow \mathcal{A} \epsilon,
$$

a geometric quantity $\mathcal{S}_{d_{F}}$ of dimension $d_{F}$ scales to

$$
\mathcal{S}_{d_{F}} \rightarrow \mathcal{S}_{d_{F}} \epsilon^{d_{F} / 2}
$$

The average coordination of tiles in the Penrose tiling is four, meaning the number of vertices is equal to the number of tiles; thus, under inflation, the total number of vertices in the infinite tiling (hence, the effective area) increases by a factor $\epsilon=\varphi^{2}$, whereas the number of vertices along a monomer membrane doubles. Hence, the fractal dimension of the monomer membrane is determined by setting $\left(\varphi^{2}\right)^{d_{F} / 2}=2$, yielding

$$
d_{F}=\frac{1}{\log _{2} \varphi} \approx 1.440 \text {. }
$$

\section{MAXIMUM MATCHINGS}

In Sec. III, we establish that Penrose tilings do not admit perfect matchings. Consequently, any dimer covering must necessarily include monomers. In this section, we proceed to find a set of maximum dimer matchings, which host the fewest possible monomers. We do so in two steps: First, in Sec. IVA we provide an algorithm for generating dimer matchings, and then in Sec. IV B we prove that the matchings generated by this algorithm indeed contain the maximum number of dimers.

\section{A. Dimer inflation algorithm}

Recall that the Penrose tiling can be constructed by an inflation procedure. It is natural to ask whether we can leverage this fact to construct dimer coverings in a similar fashion. Consider a finite section of the Penrose tiling generated by inflating a simply connected tile set a finite number of times. We can imagine decorating the seed by placing dimers on certain edges before inflation. Strictly, the procedure to achieve $n$ inflations would be to inflate $n-1$ times via the standard inflation rules, then once with the decorated inflation rules which specify the dimer positions. As an example, Fig. 8(b) shows a finite section of the Penrose tiling obtained by performing four inflations of the local empire of the $5_{A^{-}}$ vertex using the standard inflation rules of Fig. 2, followed by a final inflation using the dimer-decorated inflation rules specified in Fig. 8(b). However, this figure also illustrates a general obstruction to this procedure: namely, that it always leads to some vertices in the final covering which are covered twice by dimers (which is forbidden).

It is impossible to create a maximum matching with the full inflation symmetry of the tiles.

Proof.-This is seen most easily by observing that the three edges on the central red triangles of the thin tile become the three edges of the 6-vertex, one of which must be covered by a dimer in a maximum matching [Fig. 6(b)]. Placing a dimer on any one of these edges leads to a forbidden double cover elsewhere in the inflated pattern.

However, a simple algorithm can be applied to any forbidden dimer covering [such as that in Fig. 8(b)] to produce an allowed matching. Whenever a vertex is covered by two dimers, simply delete one. This will create a monomer neighboring the formerly double-covered vertex. Note also that monomers existed already (generated by the inflation, not the deletion) at $5_{B}$-vertices and some $5_{A}$-vertices. In the case of the $5_{B}$, this is unavoidable, as all five of the edges meeting at these vertices come from the same edge in the inflation tiles (the edge lying on the mirror axis of the thick rhombus).

This construction, which we term the dimer inflation algorithm, can be seen to place monomers as follows: 
(i) If a $5_{A^{-}}$or $5_{B^{-}}$-vertex has no 7 -vertices as secondnearest neighbors, a monomer is placed with the same bipartite charge as the 5-vertex.

(ii) If a $5_{A}$-vertex has one 7-vertex as a second-nearest neighbor, a monomer is placed with opposite bipartite charge to the 5-vertex.

(iii) If a $5_{A}$-vertex has two 7 -vertices as second-nearest neighbors, three monomers are placed with opposite bipartite charge to the 5 -vertex.

The monomers in (i)-(iii) are the only monomers placed by the dimer inflation algorithm.

Proof.-Allowing for overlaps, the local empire of the 4-vertex is sufficiently large that it can cover the entire Penrose tiling. Therefore, the inflation of the local empire of the 4-vertex can cover the inflated tiling. Figure 10(a) shows the local empire of the 4-vertex and Fig. 10(b) its inflation by the dimer-decorated inflation tiles. This tile set including its dimer decoration covers the entire tiling, and it suffices to check that the dimer inflation algorithm places only monomers according to the authority of (i)(iii), the algorithm places only monomers (and does not place anything other than monomers). A $5_{B}$-vertex
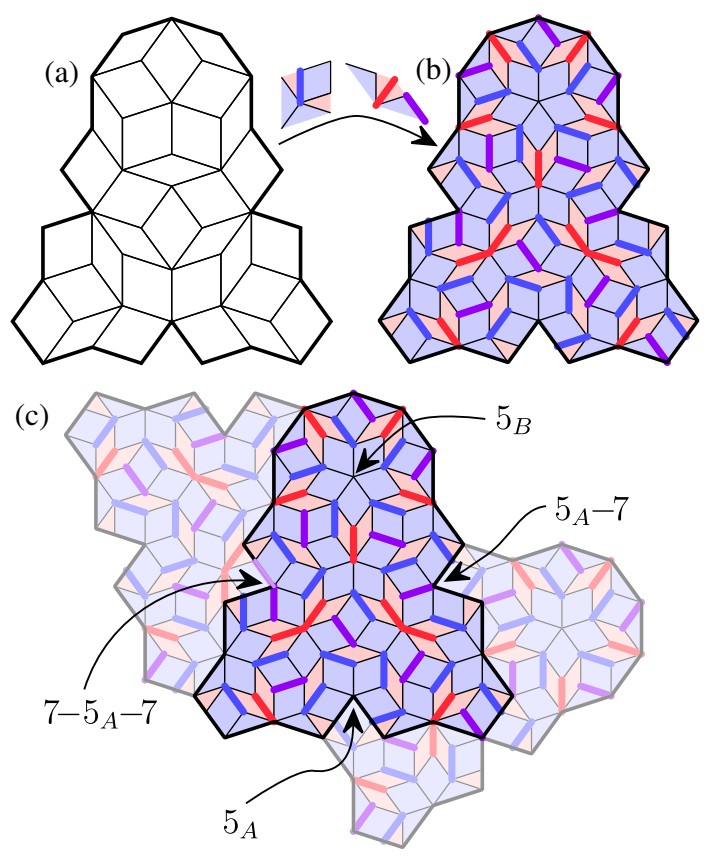

FIG. 10. Proof that the dimer inflation algorithm of Sec. IV A associates monomers with $5_{A, B}$-vertices based on how many 7 vertices they have as second-nearest neighbors [points (i)-(iii) in that section]. (a) The local empire of the 4-vertex is large enough to cover the Penrose tiling, allowing for overlaps. (b) Inflating using the dimer-decorated inflation rules leads to a set of tiles large enough to cover the inflated tiling. (c) To confirm statements (i)-(iii) about monomer placement, it is necessary to check every continuation of the boundary vertices. Two continuations are shown, with relevant features highlighted: a $5_{B}$-vertex, and $5_{A}$-vertices with zero, one, and two 7-vertices as second-nearest neighbors. appears in the tile set and hosts a monomer, in agreement with (i). All $5_{A}$-vertices appear on the boundary of the tile set upon different continuations. All possible continuations of the boundary consistent with the matching rules must be checked. Figure 10(c) shows two continuations of the tile set; the combined tile set features all combinations of $55_{A, B^{-}}$and 7 -vertices and all obey the stated monomer placement rules. We check all possible boundary completions and confirm that statements (i)(iii) hold and that no further monomers are created by the dimer inflation algorithm.

As there are 12 edges in total between the two onceinflated tiles, there are $2^{12}$ choices for dimer coverings consistent with inflation. We prove in Sec. IV B that the algorithm considered here leads to a maximum dimer matching, and so no other choice can do better. However, we find several other examples which do as well as this choice, albeit with slightly more complicated rules for removing double-dimer coverings of vertices.

\section{B. The dimer inflation algorithm produces maximum matchings}

To see that the matching generated by the dimer inflation algorithm in Fig. 8(b) is maximum, recall the following facts. First, the local empire of the 6-vertex (Fig. 11) is sufficiently large that it can cover the entire Penrose tiling, allowing for overlaps [81]. Second, monomers cannot cross monomer membranes (solid thick black edges in Fig. 11 for

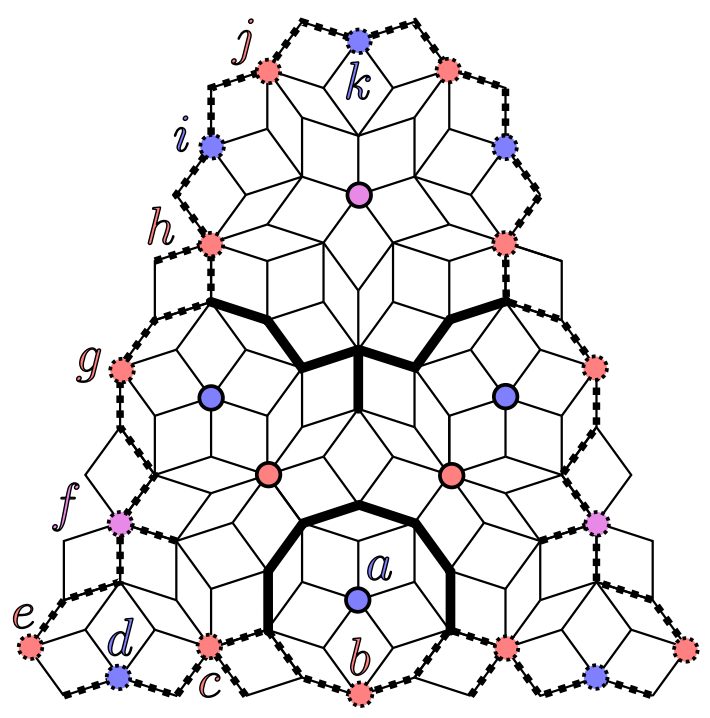

FIG. 11. The local empire of the 6-vertex with key vertices identified, used in the proof in Sec. IV B and Appendix B that the dimer inflation algorithm in Fig. 8(b) generates maximum matchings. Solid (dashed) circles indicate vertices of definite (potential) valence: red 7 -vertices, blue $5_{A^{-}}$-vertices, and pink $5_{B^{-}}$ vertices. Solid (dashed) thick black lines indicate definite (potential) segments of monomer membranes (which cannot be covered by dimers or crossed by monomers). 
even-loop segments, dashed thick black edges for potential even-loop segments). Third, the dimer inflation algorithm creates monomers as stated in points (i)-(iii) of Sec. IV A. If we can prove that no monomers placed by the algorithm are connected by augmenting paths, then the matching must be maximum. The only obstacles to augmenting paths are monomer membranes. We need to check only the relationships between the 5- and 7-vertices, rather than the monomers themselves, as the relationship between the monomers and the vertices is specified in (i)-(iii).

For example, any path connecting any two $5_{B}$-vertices must contain an even number of edges (be of even length) if it does not cross an impermeable monomer membrane. This is because each $5_{B}$-vertex has associated with it a monomer of the same bipartite charge in a maximum matching. Two of these monomers must have the same charge if they are not separated by a monomer membrane. If they had opposite charge they would be connected by an augmenting path, and the matching would not be maximum. Therefore, any two $5_{B}$-vertices connected by a path not crossing a monomer membrane must also have the same bipartite charge. As a corollary, any path connecting two $5_{B}$-vertices and not crossing a monomer membrane must itself be of even length.

In fact, not only does this result hold but so does a stronger one: Any path connecting any two $5_{B}$-vertices crosses an even (odd) number of edges if it crosses impermeable monomer membranes an even (odd) number of times. This implies not only that the $5_{B}$-related monomers within one region are of the same bipartite charge, but those within neighboring regions are of opposite bipartite charge. Consider the local empire of the 6-vertex in Fig. 11. It contains one certain $5_{B}$-vertex (pink, solid circle) in the bulk, and two potential $5_{B}$-vertices on the boundary related by a mirror symmetry (pink, dashed circles). The solid thick black lines indicate segments of the impermeable monomer membrane, and the dashed thick black lines indicate potential monomer membrane segments. As the membrane forms a closed loop, it must separate the known $5_{B}$-vertex from the two potential $5_{B}$-vertices. We see that these vertices are separated by paths of odd length. The two boundary pink potential $5_{B}$-vertices are separated by paths of even length. However, if they are both $5_{B}$-vertices, this fact resolves the ambiguity of the potential monomer membranes passing through them and confirms that they are not separated by a monomer membrane, which is correct. As the entire tiling can be constructed from this local empire, there can be no other possible relations between $5_{B}$-vertices.

To complete the proof, it suffices to prove the following statements:

(i) Any path connecting any two 7-vertices is of even (odd) length if it crosses impermeable monomer membranes an even (odd) number of times.

(ii) Any path connecting any two $5_{A, B}$-vertices, where the $5_{A, B}$-vertices have no 7 -vertices as second-nearest

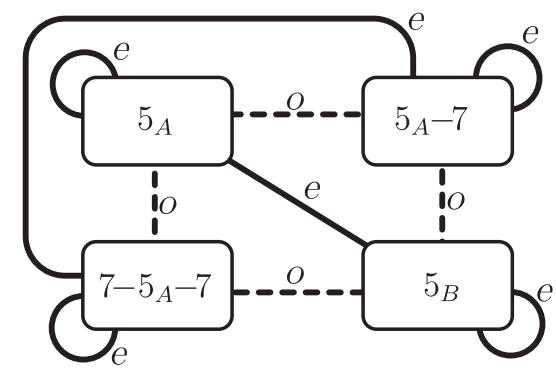

FIG. 12. The listed vertex types must be separated by paths of even ( $e$, solid lines) or odd ( $o$, dashed lines) length if the vertices are separated by an even number of monomer membranes (including zero). If the vertices are separated by an odd number of monomer membranes, $e$ and $o$ should be interchanged. The box labeled " $5_{A}$ " excludes the " $5_{A}-7$ " and " $7-5_{A}-7$ " configurations, and $5_{A}-7$ excludes $7-5_{A}-7$. See Fig. 10 and Sec. IV B.

neighbors, is of even (odd) length if it crosses impermeable monomer membranes an even (odd) number of times.

(iii) Any path connecting any 7 -vertex to any $5_{A, B^{-}}$ vertices, where the $5_{A, B}$-vertices have no 7 -vertices as second-nearest neighbors, is of odd (even) length if it crosses impermeable monomer membranes an even (odd) number of times.

The statements are summarized in Fig. 12. Note that the only monomer membrane permeable to monomers is the $4^{5}$-loop. The results are evident for the interior of the local empire of the 6-vertex; the only cases to check are therefore any (ambiguous) boundary vertices which have the potential to become one of the vertices in question.

The options are presented in Fig. 11. Red vertices are 7-vertices (solid circles indicate certain 7-vertices, whereas dashed circles indicate potential 7-vertices); blue vertices are (potential or actual) $5_{A}$-vertices; pink vertices are (potential or actual) $5_{B}$-vertices. The verification that every case works is lengthy but straightforward and appears in Appendix B. In all possible cases, the three listed requirements are fulfilled, and the dimer inflation algorithm given in Sec. IVA generates maximum matchings.

\section{MONOMER DENSITIES IN MAXIMUM MATCHINGS}

Having presented in Sec. IV an algorithm for generating maximum matchings of the Penrose tiling, we proceed to analytically calculate (in Sec. VA) and numerically check (in Sec. V B) the density of monomers in any maximum matching.

\section{A. Analytic calculation of the minimal monomer density}

The results of Sec. IV reveal that we can associate monomers with $5_{A}$-vertices, $5_{B}$-vertices, and 7 -vertices. 
TABLE I. The vertices in the dart-kite $P 2$ tiling (Fig. 3); their equivalents in the rhombic $P 3$ tiling; their inflations in $P 3$; their relative frequencies; simplified forms of the frequencies, making use of the defining equation of the golden ratio $\varphi^{2}=\varphi+1$.

\begin{tabular}{lcccc}
\hline \hline Dart kite & Rhombus & Inflation & Frequency & Simplified \\
\hline Queen & $3_{A}$ & 7 & $\varphi^{3}\left(\varphi^{4}-1\right)$ & $11 \varphi+7$ \\
Deuce & $3_{B}$ & $3_{A}$ & $\varphi^{5}\left(\varphi^{4}-1\right)$ & $29 \varphi+18$ \\
King & 4 & 6 & $\varphi^{2}\left(\varphi^{4}-1\right)$ & $7 \varphi+4$ \\
Star & $5_{A}$ & $5_{B}$ & $\varphi^{4}$ & $3 \varphi+2$ \\
Sun 5 & $5_{B}$ & $5_{A}$ & $\varphi^{2}$ & $\varphi+1$ \\
Jack & $5_{C}$ & 4 & $\varphi^{4}\left(\varphi^{4}-1\right)$ & $18 \varphi+11$ \\
Sun 4 & 6 & $5_{A}-7$ & $\varphi^{4}-1$ & $3 \varphi+1$ \\
Sun 3 & 7 & $7-5_{A}-7$ & $\varphi\left(\varphi^{4}-1\right)$ & $4 \varphi+3$ \\
Ace & $\cdots$ & $\cdots$ & $\varphi^{6}\left(\varphi^{4}-1\right)$ & $47 \varphi+29$ \\
\hline \hline
\end{tabular}

The monomers are not constrained to sit at specific locations relative to these vertices, as they can move to any vertices to which they connect via alternating paths. However, we are able to use the association between monomers and vertices implied by the dimer inflation algorithm [points (i)-(iii) in Sec. IV A] to establish the density of monomers in maximum matchings of the Penrose tiling.

Table I lists the relative frequencies of each vertex type in the Penrose tiling $[53,85,86]$. If we consider not just the vertex and its inflation, but the local empires of each, certain facts become evident. A $5_{A, B}$-vertex with no 7 -vertices as second-nearest neighbors is created by inflating a $5_{B, A}$-vertex; this pattern is associated with one

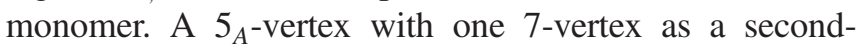
nearest neighbor is created by inflating a 6-vertex; this pattern is also associated with one monomer. Finally, a $5_{A^{-}}$ vertex with two 7-vertices as second-nearest neighbors is created by inflating a 7-vertex; this pattern is associated with three monomers. New vertices are also generated between existing vertices upon inflation, but inspecting the inflated vertices in Fig. 3 reveals that only $3_{B^{-}}$and $5_{C^{-}}$ vertices appear in this way.

Putting the results together, we find that the density of monomers $\rho_{\text {monomer }}$ in the tiling is

$$
\rho_{\text {monomer }}=\frac{f\left(5_{A}\right)+f\left(5_{B}\right)+f(6)+3 f(7)}{\varphi^{2} \sum_{v \in \text { vertices }} f(v)},
$$

where $f(v)$ is the relative frequency with which a $v$-vertex appears (see Table I). The factor of $\varphi^{2}$ in the denominator arises because it is the inflated pattern which is to be compared to, and the number of all vertices increases by $\varphi^{2}$ under inflation in the infinite tiling. Substituting the values from Table I, we find the result for the monomer density:

$$
\rho_{\text {monomer }}=81-50 \varphi \text {, }
$$

where the simplified result follows from repeated use of the defining equation of the golden ratio, $\varphi^{2}=\varphi+1$.

\section{B. Numerical confirmation of the minimal monomer density}

In order to confirm the analytic result of the previous section, we carry out numerical calculations of the monomer densities in finite-size sections of the Penrose tiling. To generate the sections, we carry out up to 13 inflations on seed tile configurations using the inflation rules of Fig. 2. We use as the seeds only the two basic rhombuses, thick and thin. Any other seed is simply a combination of these, differing only on the boundary.

In Fig. 13, we show the numerically obtained monomer densities (number of monomers in the finite system divided by the total number of vertices in the system). The inflation method can leave stray twigs on the boundary of the system; we prune these before finding maximum matchings. We consider two possible methods of placing monomers: the dimer inflation algorithm outlined in Sec. IV A (solid red squares for inflations of the thick tile, solid blue circles for inflations of the thin tile) and the Hopcroft-Karp algorithm [62]. We test the Hopcroft-Karp result against an

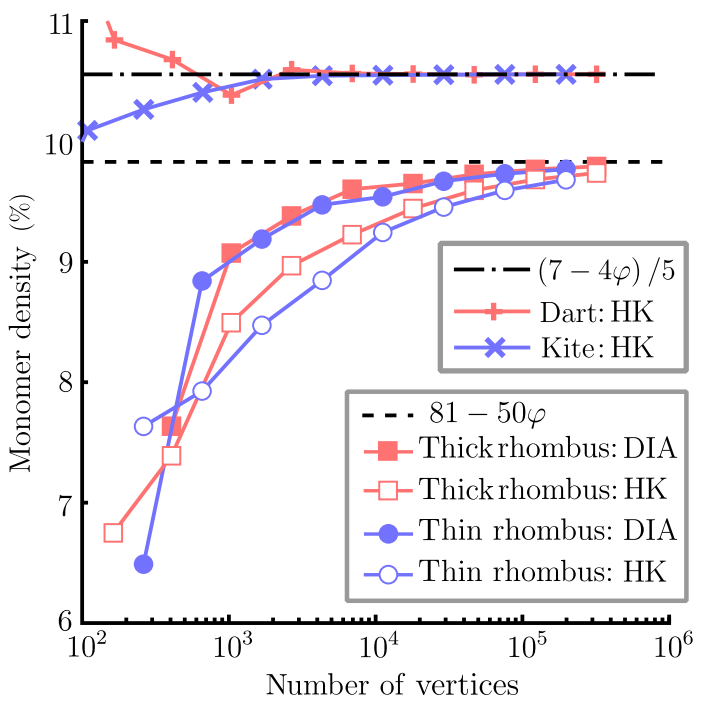

FIG. 13. Numerical results for the density of monomers in finite sections of the Penrose tiling. To achieve results with the least possible bias, we consider inflations of each basic tile: the thick (red squares) and thin (blue circles) rhombuses for $P 3$ and the dart (red crosses) and kite (blue crosses) for P2 (see Sec. VII). For the rhombic tiling, solid symbols correspond to systems in which monomers are placed according to the dimer inflation algorithm (DIA) of Sec. IVA, whereas hollow symbols correspond to systems in which maximum matchings are found using the Hopcroft-Karp algorithm (HK, also used for the dart-kite tiling). The analytical results of $81-50 \varphi \approx 0.098$ for the rhombic $P 3$ tiling and $(7-4 \varphi) / 5 \approx 0.106$ for the dart-kite $P 2$ tiling are shown by the black dashed lines. 
alternative maximum matching method, the Eppstein algorithm, and obtain identical results.

Differences between the numerical and analytical results may be attributed both to the usual boundary effects in a finite system and to the impossibility of reproducing an irrational number as a ratio of two integers. In Fig. 13, we see that both methods of monomer placement lead to monomer densities tending rapidly to the analytic value (note the logarithmic scale on the horizontal axis). The dimer inflation algorithm is less affected by the boundaries than the Hopcroft-Karp matching. The dimer inflation algorithm incurs errors in monomer placement only within one vertex from the boundary when the boundary artificially decreases the valence of the vertex. The HopcroftKarp method, on the other hand, can take advantage of augmenting paths connecting bulk monomers with monomers artificially created by the boundary, and these paths can be of arbitrary length provided they are not constrained by monomer membranes.

\section{THE MAXIMUM MATCHING MANIFOLD IS CONNECTED BY MINIMAL MONOMER MOVES}

In this section, we demonstrate that, starting from one maximum matching, all others can be reached by minimal monomer moves without passing through nonmaximum matchings. The set of maximum matchings therefore forms a connected manifold.

To motivate this idea, consider a general bipartite graph which admits a perfect matching. Deleting a dimer from such a matching creates two neighboring monomers of opposite bipartite charge. In physical models, such a paircreation process is expected to be energetically costly. Assigning an energy to pair creation, the set of perfect matchings constitutes the degenerate set of classical ground states of the system. Starting from one perfect matching, if all others can be accessed by reconfiguring dimers in a physical manner (discussed shortly) while remaining within the set of perfect matchings, the set of degenerate classical ground states forms a connected manifold [87]. This fact is a necessary but not sufficient condition for the system to be ergodic, meaning that the time spent under dynamical evolution in a given volume of phase space of equal-energy microstates is proportional to the volume itself, or, equivalently, that all accessible microstates are sampled equally over sufficiently long timescales [88]. In the present work, we are not specializing to a particular energy function or physical model, and the results can be considered to be in the zero-temperature limit.

The sort of features which could disconnect a set of matchings might be, for example, the presence of noncontractible system-spanning loops under periodic boundary conditions. These loops can disconnect the phase space into topological sectors unreachable from one another by local (i.e., physical) moves [4]. Before considering the case of the Penrose tiling, it is instructive to continue to consider an arbitrary bipartite graph admitting multiple perfect matchings. In the case that only one topological sector exists, all perfect matchings can be enumerated by the following process: Starting from one perfect matching (which can be found, for instance, by the Hopcroft-Karp algorithm), another can be generated by augmenting an alternating cycle. In fact, all perfect matchings can be enumerated by augmenting all alternating cycles in a given perfect matching [89]. The following physical analogy motivates such moves: Deleting one dimer on the cycle creates a monomer-antimonomer pair. The monomer can then hop around the cycle via minimal monomer moves and reannihilate with the antimonomer, with the net effect being the desired augmentation. The analogy is imperfect, however, as the intermediate stages are not contained within the set of perfect matchings. In quantum dimer models, they can be considered virtual processes.

Now consider the case of a finite bipartite graph which does not admit a perfect matching. Starting from a maximum matching, we further restrict to the case that every alternating cycle connects to at least one monomer via alternating paths. All maximum matchings can now be enumerated by identifying and augmenting all alternating cycles, plus all alternating paths (by definition, each alternating path terminates on precisely one monomer) [89]. The physical analogy holds more closely in this case. Augmenting an alternating path can be achieved simply by hopping the monomer along the path with minimal monomer moves. Augmenting an alternating cycle can be achieved by hopping a monomer to any vertex on the cycle, hopping the monomer around the cycle, then hopping the monomer back to its initial vertex along the same path it took to the cycle. In this process, the only change to the dimer configuration is to augment the alternating cycle, since dimers on the path connecting the starting vertex to the cycle are returned to their initial configuration when the monomer retraces its steps.

Since no pair creation occurs, these processes stay within the set of maximum matchings. The restriction that all alternating cycles connect via alternating paths to monomers means that it is always possible to find a monomer to carry out such a move. A simple example of a graph violating this condition is to add to this example a second graph, disconnected from it, which itself admits multiple perfect matchings: Therefore, the total graph still does not admit a perfect matching, but the perfectly matched region still requires the unphysical virtual processes to augment its alternating cycles.

Recall that the Penrose tiling divides into monomer regions bounded by monomer membranes. At most, two monomer membranes (i.e., one monomer region) can cross the entire system. The others are closed with $D_{5}$ symmetry. Since monomer membranes can never host dimers in 
maximum matchings, we can consider each monomer region separately. Every monomer region contains an excess of bipartite charge.

Proof.-This can be seen (admittedly in a slightly roundabout way) from the fact that (i) the dimer inflation algorithm generates maximum matchings with monomers on every $5_{B}$-vertex, (ii) every monomer region contains a $5_{B}$-vertex (since the local empire of the 6-vertex contains $5_{B}$-vertices on both sides of the impermeable membrane and covers the entire tiling), and (iii) the total number of monomers in a monomer region is equal to the monomer region's net bipartite charge.

All vertices with the same bipartite charge as their monomer region connect via alternating paths to monomers.

Proof.-We just proved that every monomer region contains at least one monomer. To see that every vertex within a monomer region (where the vertex has the same bipartite charge as the region) connects to a monomer via an alternating path, consider the following argument. Pick an arbitrary vertex $v_{0}$ within the monomer region with the same charge as the monomers in that region. If the vertex hosts a monomer, we are done. If not, the vertex must be covered by a dimer. Consider the vertex at the other end of the dimer, $v_{1}$. All the other edges of $v_{1}$ must be uncovered. Consider these edges and the vertices $v_{2}^{i}$ (where $i+1$ is the valence of $v_{1}$ ) to which they connect. If any of $v_{2}^{i}$ hosts a monomer, this monomer then connects via an alternating path $v_{2}^{i}-v_{1}-v_{0}$ to the original vertex $v_{0}$, and we are done. If not, each of $v_{2}^{i}$ is covered by precisely one dimer. By iterating this process, a monomer must eventually be reached.

Since the Penrose tiling is bipartite and divides into closed regions in which every alternating cycle connects via an alternating path to a monomer, the set of maximum matchings forms a manifold connected by minimal monomer moves.

It is not true that any monomer within a monomer region connects via an alternating path to any given vertex with the same bipartite charge as the region. In terms of minimal monomer moves, a rearrangement of the remaining monomer positions may in general be necessary to facilitate a monomer reaching a given vertex. Figure 8(a) shows two monomer regions of the Penrose tiling obtained from Fig. 7 by removing edges which can never be covered by dimers in maximum matchings, then by removing the largest connected component of the resulting disconnected graph (the outermost monomer region in Fig. 7). One monomer is highlighted with an arrow and gold cross; the set of vertices connected to this monomer by alternating paths is highlighted in gold. This is the set of vertices the monomer can reach via minimal monomer moves, holding all other monomers fixed. The dark gray vertices are even-valence vertices before disconnection and form the unreachable monomer membrane which bounds the monomer region. Other vertices intermingled with the gold vertices are of the wrong bipartite charge and also cannot be reached. However, there are many more vertices within the region which are legitimate sites for occupation and simply cannot be reached. The obstacle is not directly provided by other monomers, although these can also in principle form obstructions; instead, it is the dimer network. By moving monomers other than the crossed monomer, it is possible to rearrange the dimer network to allow the crossed monomer to reach any vertex.

\section{CLASSICAL DIMERS ON OTHER PENROSE-LIKE TILINGS}

All rhombic Penrose tilings are locally isomorphic to one another. Different decorations of the tiles can lead to alternative Penrose tilings in different local isomorphism classes. As the resulting graph connectivity changes under such decorations, the properties of maximum matchings may also change. In order to place our results of the rhombic Penrose tiling in a more general context, in this section we consider dimer coverings of other twodimensional Penrose-like tilings.

\section{A. Other Penrose tilings}

The $P 3$ rhombic Penrose tiling is the third to be identified. Earlier versions include the dart-kite tiling $P 2$, and the original pentagonal tiling $P 1$, which has four different tile types. Figure 2 shows decorations of the $P 3$ tiles which lead to the $P 1$ and $P 2$ tilings [90]. Since one of the $P 1$ tiles is a pentagon, $P 1$ necessarily contains cycles of odd length, and the corresponding graph is therefore not bipartite. For this reason, we do not discuss it further. In this section, we consider the dimer-covering properties of the dart-kite $P 2$ tiling and provide another simple decoration of $P 3$ which trivially results in a perfect matching.

\section{The Penrose dart-kite (P2) tiling}

The Penrose $P 2$ tiling consists of two tiles referred to by Penrose as the dart and kite. Figure 2 shows how to derive a $P 2$ tiling by decorating the rhombic tiles of $P 3$. Alternatively, $P 2$ can be obtained by its own inflation rules. As both tiles have four sides, the graph is again bipartite. A finite region of the $P 2$ tiling is shown in Fig. 14. $P 2$ features nine vertex types. Following Ref. [53], we term them the deuce, jack, queen, king, ace, star, and suns 3-5 (as with $5_{A}$ and $5_{B}$ in the rhombic tiling, the different sun vertices are distinguished by their neighboring tiles; the number indicates the number of dart tiles pointing out from the sun). Except the ace, all vertices are in one-to-one correspondence with vertices in the rhombic tiling; the correspondence is given in Fig. 3 and Table I. Inspecting the local empires of each vertex reveals an interesting property: Each vertex belongs to precisely one-bipartite sublattice. The suns, deuce, and jack form the entirety of one sublattice [blue vertices in Fig. 14, and the star, queen, 


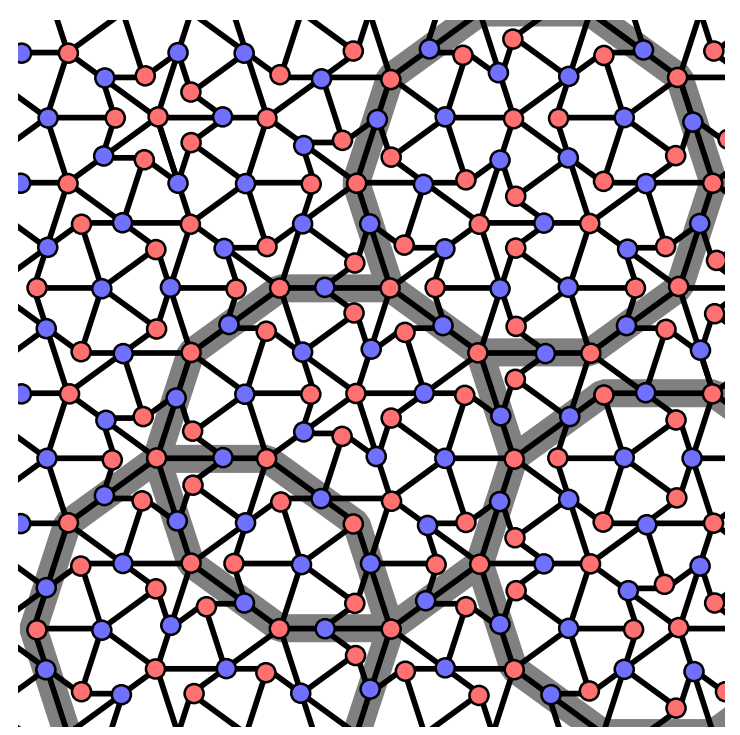

FIG. 14. A region of the Penrose dart-kite $(P 2)$ tiling. The vertices (Fig. 3) divide into bipartite sublattices: star-queen-kingace (red) and deuce-jack-suns (blue). Dark gray decagons indicate cartwheels, regions which can cover the infinite tiling allowing for overlaps [81].

king, and ace form the other (red)]. This property of $P 2$ is in contrast to $P 3$ in which each vertex appears in both sublattices. The $P 2$ tiling admits a covering (allowing for overlaps) by a set of tiles known as a cartwheel $[54,81,90]$. Some cartwheels are highlighted in Fig. 14.

Figure 15 shows a maximum matching of the same region obtained using the Hopcroft-Karp algorithm. A number of monomers appear all on the same bipartite sublattice. Inspecting the vertex frequencies in Table I

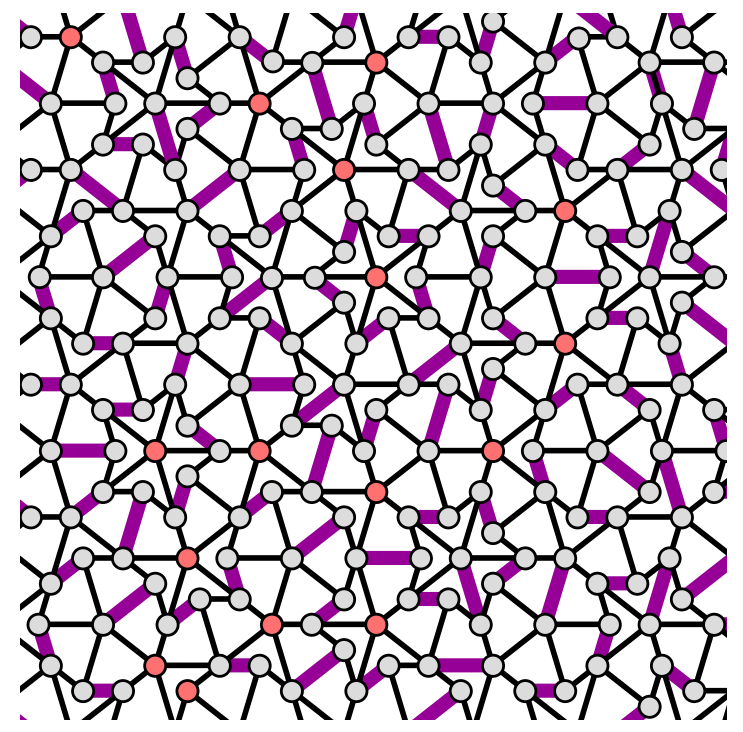

FIG. 15. A maximum matching of the Penrose dart-kite (P2) tiling in Fig. 14. Monomers are shown in red. All lie on the same bipartite sublattice. reveals an imbalance in the relative frequencies of vertices in the two sublattices: In the infinite tiling, the star-queenking-ace sublattice contains more vertices than the sundeuce-jack sublattice. A perfect matching is therefore impossible, as the lower bound on the monomer density is given by the excess density of one sublattice over the other. In fact, this lower bound is the true monomer density. To see this, it suffices to prove that monomers on the starqueen-king-ace sublattice can reach any vertex on that sublattice: If this is the case, the sun-deuce-jack sublattice must be perfectly matched, otherwise, the total number of monomers could be decreased by moving a monomer on the star-queen-king-ace sublattice next to a monomer on the sun-deuce-jack sublattice, then annihilating both. Therefore, if the monomers present on the star-queen-kingace sublattice can reach any vertex of that sublattice, then the sun-deuce-jack sublattice is perfectly matched, and the matching contains the minimum number of monomers.

Any vertex on the star-queen-king-ace sublattice can be reached by a monomer.

Proof.-By considering an arbitrary maximum matching of the cartwheel with each of its continuations, any vertex on the star-queen-king-ace sublattice can be seen to connect via an alternating path to a monomer. As every vertex in the infinite tiling appears within a cartwheel, every vertex on the infinite star-queen-king-ace sublattice connects to a monomer by an alternating path, and all vertices on this sublattice can be reached by a monomer.

Therefore, the monomer density on $P 2$ is precisely given by the imbalance of vertices between the two sublattices. This density can be found as follows:

$$
\rho_{\text {monomer }}=\frac{\sum_{v \in A} f(v)-\sum_{v \in B} f(v)}{\sum_{v \in A} f(v)+\sum_{v \in B} f(v)},
$$

where $f(v)$ is the relative frequency with which vertex $v$ appears (see Table I), $A$ denotes the set of vertices on the star-queen-king-ace bipartite sublattice, and $B$ denotes the set of vertices on the deuce-jack-sun sublattice. The result simplifies to

$$
\rho_{\text {monomer }}=(7-4 \varphi) / 5 \text {. }
$$

We again confirm this result numerically by employing the Hopcroft-Karp algorithm to find maximum matchings of successively larger finite-sized regions created by inflating the two basic tiles. The result shown in Fig. 13 shows a rapid convergence toward the analytic result, which is valid in the thermodynamic limit.

We note that this result should be sharply contrasted with the case of the rhombic tiling: There, in the thermodynamic limit there is no net imbalance in the vertices of any sublattice; the overall tiling is, as we show, charge neutral. Instead, there the presence of a finite density of monomers is a consequence of the impermeability of monomer 
membranes that "cage" positively and negatively charged monomers into separate regions and prevent them from annihilating. The fact that the monomer density in the kitedart tiling precisely matches the excess of one sublattice over the other in the thermodynamic limit shows that such caging does not occur here.

\section{Decorated Penrose tilings}

The different Penrose tilings $P 1-P 3$ can be derived from one another as decorations of the tiles. $P 1$ is nonbipartite, $P 2$ is bipartite and features charged maximum matchings, and $P 3$ is bipartite and features charge-neutral maximum matchings. It is natural to ask whether a decoration of the tiles is possible, which leads to a bipartite graph admitting perfect matchings. In fact, such decorations are simple to design, with one option shown in Fig. 16. Each tile of $P 3$ has been decorated with edges, vertices, and dimers, in such a way that a perfect matching is present by construction [91]. All vertices of $P 3$ appear in the region shown, and it can be seen that the number of graph edges enclosing a valence- $v$ vertex is $2 v$. As this number is even, and the only other cycles (appearing within the tiles) are of length four, the graph is bipartite. Some of the edges of the graph are allowed to curve for ease of drawing.

\section{B. Eightfold and 12-fold Penrose-like tilings}

By considering various decorations of Penrose tilings, we find a wide range of behaviors of classical dimer models. In order to establish the generality of these results, in this section we briefly consider classical dimers on other two-dimensional Penrose-like tilings.

All known examples of physical quasicrystals have five-, eight-, ten-, or 12-fold rotational symmetry and feature symmetries related to quadratic irrational PV numbers $[50,55,58]$. In the present paper, we construct Penrose-like tilings using inflation rules. An alternative construction method is based on the use of Ammann bars, decorations of the tiles with straight line segments such that valid tile

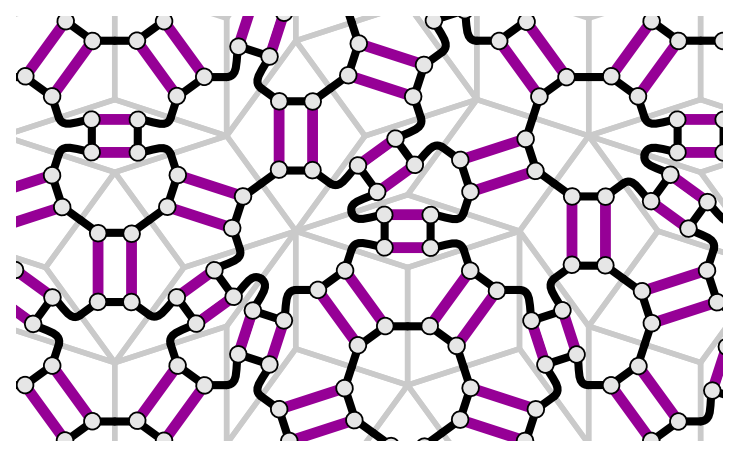

FIG. 16. The rhombic Penrose tiles (light gray) decorated with edges (black), vertices, and dimers (purple) such that each tile of the same type is identical, and the resulting bipartite graph admits a perfect matching. configurations lead to infinite sets of straight lines [32,54]. Reference [56] identifies that there are only six possible two-dimensional Penrose-like tilings which have the minimal set of one-dimensional Ammann decorations compatible with their orientational symmetries. The authors of that paper provide a method of constructing the tilings from the Ammann bars. The construction leads to tilings made up of small numbers of inequivalent convex tiles (as in $P 3$, which has two tiles). The six two-dimensional Penrose-like tilings resulting from this construction are as follows: the rhombic Penrose tiling (with a tenfold symmetric diffraction pattern, and PV number $\varphi$ ), tilings $8 A$ and $8 B$ with eightfold symmetric diffraction patterns and $\mathrm{PV}$ number $1+\sqrt{2}$ (the silver ratio), and tilings $12 A-12 C$ with 12 fold symmetric diffraction patterns and PV number $2+\sqrt{3}$.

We save a full analysis of the behavior of dimer matchings in all of these cases for future work. However, we are able to make a number of comments. Case $8 A$ is better known as the Ammann-Beenker tiling $[32,90]$. In upcoming work, we prove that this case admits perfect matchings [92]. Turning to case $8 B$, we are able to find a perfect matching of a large finite region, and we conjecture that the infinite tiling also admits perfect matchings.

Case $12 \mathrm{~A}$ is better known as the Socolar-Taylor tiling $[56,93]$. It contains three tiles with angles which are multiples of $2 \pi / 12$. Creating a maximum matching of a finite region, we find that monomers of both charges necessarily exist and are trapped by uncrossable membranes as in the rhombic Penrose tiling. A sample of this matching is shown in Fig. 17. The existence of any monomer unable to reach the boundary of a finite region, in any maximum matching, is sufficient to prove that perfect matchings do not exist for the infinite tiling. The matching in Fig. 17 contains one blue monomer and four red monomers; in lighter colors are vertices which connect to these monomers via alternating paths and which can therefore be reached under minimal monomer moves. While the matching itself is arbitrary, the set of vertices which can be reached by monomers is independent of matching [94]. Monomer membranes separate regions of opposite bipartite charge, as in the rhombic Penrose tiling, and again appear to follow chains of 4- and 6-vertices interspersed with 5-vertices. Unlike the Penrose tiling, however, the Socolar-Taylor membranes are able to branch. Branchings appear to occur at double-width membrane segments formed from two 4-vertices appearing back to back across a thin tile. These double-width segments are then able to separate regions of the same bipartite charge (we use two different colors of light red to indicate these distinct regions).

Cases $12 B$ and $12 C$ are similarly unable to admit perfect matchings and feature both single- and double-width membranes as in $12 \mathrm{~A}$. Whereas all monomer regions we 


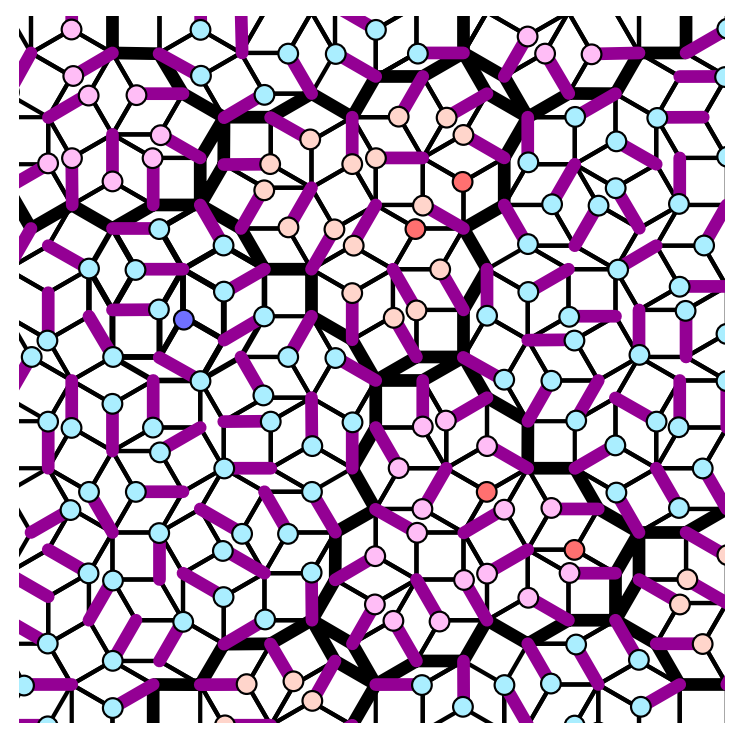

FIG. 17. A maximum matching of the Socolar-Taylor tiling $[56,93]$. Purple edges indicate dimers; the dark blue (four dark red) vertices indicate monomers, while light blue (light red) vertices connect to blue (red) monomers via alternating paths (note the two distinct colors of light red, indicating distinct monomer regions; the separation of the different blue regions is clear). Monomer membranes are identified with thick black lines.

identify in $12 \mathrm{~A}$ contain a net imbalance of charge, as in the Penrose tiling, cases $12 B$ and $12 C$ additionally feature perfectly matched islands enclosed by membranes. A maximum matching of a region of $12 B$ is shown in Fig. 18.

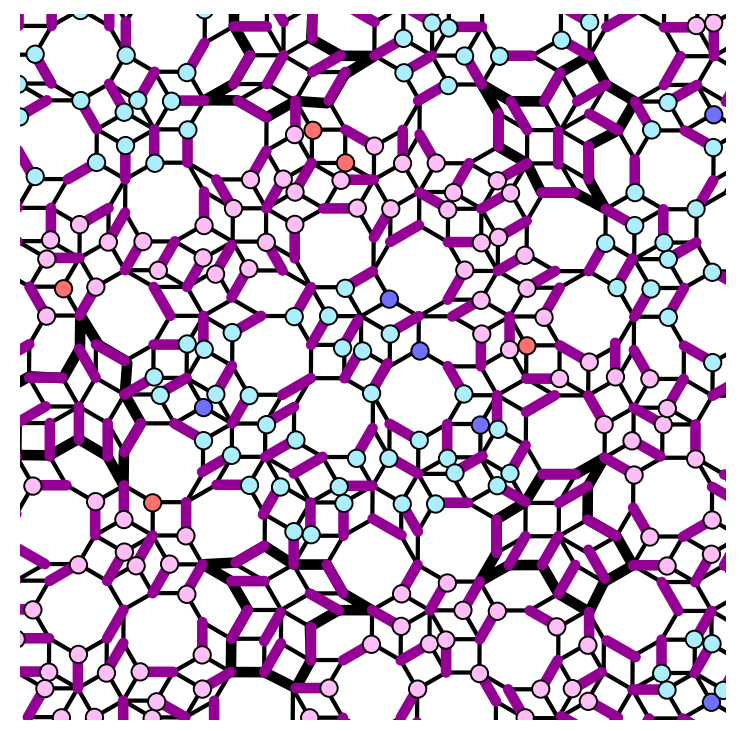

FIG. 18. A maximum matching of the Boyle-Steinhardt $12 B$ tiling [56]. Colors as in Fig. 17. Note the existence of perfectly matched regions within monomer membranes, thick black lines. These resemble manta rays converging on a coral reef at the center. These perfectly matched regions appear to be unique to the $12 B$ and $12 C$ tilings.

\section{CONCLUDING REMARKS}

In this paper, we consider dimer coverings of the Penrose tiling considered as a graph. We find that a perfect matching is not possible, but we identify various properties of maximum matchings, which are those that have the largest possible number of dimers (smallest number of monomers). We devise a method of generating these maximum matchings using the properties of the Penrose tiling (the dimer inflation algorithm) and use this to show that the density of monomers in such matchings is $81-50 \varphi$. These monomers exist in closed monomer regions bounded by loops of second-nearest-neighbor even-valence vertices. These loops are fractal objects that we dub monomer membranes, which the monomers cannot cross. Each monomer region has an excess of one or other bipartite charge equal to the number of monomers it contains in maximum matchings. Regions on opposite sides of a membrane have opposite net bipartite charge.

We note that an immediate physical application of our results is as a model for adsorbed atoms and molecules on the surfaces of quasicrystals, already known to lead to a variety of exotic structures [95-97]; it is straightforward to translate our analysis into a series of statements about such structures. However, as we note in the Introduction, the dimer model can be used to study a wide range of phenomena, and therefore, we might anticipate various other possible applications, as we now sketch.

First, we note that the physics of local constraints can drive a variety of rich phenomena even at the classical level. Perhaps the most famous recent example is the study of condensed-matter analogs of magnetic monopoles [98100]. Although they have eluded detection as fundamental particles, various experiments have indicated that they may emerge as collective excitations in the spin-ice materials dysprosium titanate and holmium titanate $\left(\mathrm{A}_{2} \mathrm{Ti}_{2} \mathrm{O}_{7}\right.$ with $A=\mathrm{Dy}$, Ho) [98]. In these materials, the magnetic rareearth ions inhabit a pyrochlore lattice of corner-sharing tetrahedra [101]. By an appropriate choice of local spin axes, the low-energy physics may be approximately captured by that of a nearest-neighbor Ising antiferromagnet. In any classical ground state, two of the four spins on a tetrahedron point into the center and two point out. Since there are six possible configurations per tetrahedron, the result is a macroscopically degenerate ground state characterized by the local two-in, two-out ice-rule constraint [102]. This situation can be viewed as Gauss's law for an emergent gauge field, but it is also linked to the local magnetization since each spin is a magnetic dipole. The lowest-energy excitation out of the ice-rule manifold consists of a single spin flip, which creates a three-in one-out tetrahedron neighboring a one-in three-out tetrahedron. Upon coarse graining by summing the divergence of the magnetization over each tetrahedron, the excitation appears as a neighboring source and sink of magnetization termed a monopole and antimonopole (the analog to 
monomers and antimonomers in the case of the dimer model). Subsequent spin flips allow the monopole and antimonopole to move apart. A Dirac string of flipped spins connects monopole-antimonopole pairs analogous to augmenting paths in the Penrose tilings considered here. In the spin-ice setting, statistical fluctuations between different spin configurations make the precise location of the string ambiguous (except with reference to a chosen starting state), giving a classical analog of the underlying gauge theory [99]. The strings also lead to an effective Coulomb law interaction between monopoles. This picture is a clear demonstration of the idea of fractionalization, albeit in a classical context, and its parallels to dimer models are clear. Our results suggest that classical frustrated magnets in quasiperiodic systems should host a similarly rich set of phenomena.

Quantum fluctuations between classically degenerate spinice configurations-generated, for instance, by transversefield terms beyond the Ising limit — can give rise to a quantum gauge theory in its Coulomb phase, with a gapless "photon" collective mode. In such a phase often termed a threedimensional $U(1)$ quantum spin liquid, the monopoles are emergent gapped quasiparticles, which carry a $U(1)$ charge and exhibit a Coulombic interaction. The search for such quantum spin ices remains an active field of research $[7,103]$.

Similarly, endowing dimer configurations with dynamics - the simplest of which is a resonance that augments the elementary alternating cycle on a single four-site plaquette-yields a quantum dimer model. On periodic bipartite lattices in three dimensions, such dimer models are also known to exist in a Coulomb liquid phase $[4,10]$; here, the photon is a collective mode of the dimers, whereas the monomers are gapped gauge-charged objects (similar to the monopoles in spin ice). However, on periodic nonbipartite lattices in any dimension, quantum dimer models lead to fully gapped dimer liquid phases with a discrete gauge structure and topological order $[3,4]$. The situation is more subtle on two-dimensional periodic bipartite lattices, as in the case of the original square-lattice quantum dimer model [1]. On such lattices, although classical dimers exhibit power-law correlations, the corresponding quantum Coulomb liquid phase is generically destroyed by instanton effects [104]. Such effects lead to the formation to a variety of dimer crystal phases, in which the emergent gauge field is confined. Deconfinement (as in the Coulomb phase) survives only at critical points between these crystal phases. However, more careful analysis of the effective theory near such transitions reveals that lattice effects can have a significant impact on their properties $[105,106]$. If the incipient crystalline order is incommensurate with the underlying periodic lattice, the dimer model remains gapless, and the collective mode survives. The interplay between the different possible dimer crystal orders and the lattice leads to a devil's staircase of gapped commensurate crystals interleaved with gapless incommensurate ones- $\mathrm{a}$ phenomenon dubbed Cantor deconfinement [105]. This situation provides one obvious motivation to study quantum dimer models on lattices, such as the Penrose tiling, where any crystalline order is likely to be frustrated.

The presence of a finite local density of monomers (but vanishing net monomer charge) suggests a route to evading the effects of instantons in a quantum extension of the present model: The presence of dynamical gauge-charged matter is known to mitigate their influence [107,108]. Intriguingly, the presence of monomer membranes that are impermeable to gauge charge suggests a rather unusual phase structure that blends aspects of both confinement and deconfinement. The presence of fractal structures that constrain the low-energy dynamics also bears a family resemblance to the physics of fractons, immobile quasiparticles that appear as low-energy excitations in certain translation-invariant Hamiltonian models [109-112], which may be viewed as end points of fractal objects. The immobility of fractons and their glassy dynamics $[109,113]$ is closely related to the properties of simple classical spin models with kinetic constraints [114]. While the precise connection between our work, the physics of fracton models, and these related physical systems remains unclear, we flag this as an interesting avenue for further study.

The various lines of investigation that we suggest above will clearly be advanced by the development of a convenient coarse-grained description of the Penrose dimer model. For dimer models on 2D periodic lattices, such a description is provided by a mapping to a so-called height model that parametrizes dimer configurations in terms of configurations of a two-dimensional surface or height field. A conventional scalar height model cannot readily be defined on the Penrose tiling, owing to the variation in valence of the various vertices. Suitable generalizations may exist which achieve the same result.

In a different vein, it would be interesting to determine which of the properties we demonstrate in the Penrose tiling carry over to other Penrose-like tilings, the members of the noncrystallographic Coxeter groups [56,115]. There is an infinite number in 1D (e.g., the Fibonacci quasilattice $[32,58,116])$, six in 2D (considered in Sec. VII), five in 3D (with the point group symmetries of the icosahedron and dodecahedron), one in 4D (with the point group symmetry of the 600 cell), and none in dimension five or higher. All can be generated by inflation rules, matching rules, and by a cut-and-project method from higher dimensions, suggesting that similar methods to those we develop here may be extended to those cases. The three-dimensional cases in particular would be interesting candidates for physically relevant systems exhibiting topological order. We also hope that the present physically motivated study of the Penrose tiling may open up new directions for studying the fascinating mathematical properties of these tilings, such as their three colorability $[32,54,117]$. 
Part of the interest in dimer coverings of graphs lies in their potential relevance to the problem of discretizing conformal field theories governing critical systems $[20,118,119]$. As Penrose-like tilings lack the discrete translational invariance of periodic lattices but instead feature a discrete scale invariance, they seem a natural subject of study from this perspective. Indeed, in the special case of conformal quasicrystals, which form the boundary of regular tilings of hyperbolic space, the structures have invariance under discretized Weyl transformations and so can be considered to host a full conformal invariance [120].

We anticipate that the themes we explore here will prove relevant to understanding the range of emergent strongly correlated phenomena possible in quasicrystals. Our aim in presenting these results has been to lay the foundations for future investigations of new and unconventional forms of classical and quantum order possible in these systems.

\section{ACKNOWLEDGMENTS}

We thank Sounak Biswas, Claudio Castelnovo, Kedar Damle, Nick Jones, Jerome Lloyd, Roderich Moessner, and Jasper van Wezel for useful discussions. F. F. acknowledges support from the Astor Junior Research Fellowship of New College, Oxford. S. H. S. is supported by EPSRC Grants No. EP/N01930X/1 and No. EP/S020527/1. S. A.P. acknowledges support from the European Research Council under the European Union Horizon 2020 Research and Innovation Programme (Grant Agreement No. 804213-TMCS), and EPSRC Grant No. EP/S020527/ 1. Statement of compliance with EPSRC policy framework on research data: This publication is theoretical work that does not require supporting research data.

\section{APPENDIX A: PROOFS ON MONOMER MEMBRANE IDENTIFICATION AND PROPERTIES}

In this Appendix, we provide proofs for the statements used to identify and study monomer membranes in Sec. III A.

\section{Neighbor structure of even-valence vertices}

We first prove the first statement in Sec. III A: namely, that any even-valence vertex in the Penrose tiling (a 4vertex or 6-vertex) has no even-valence neighbors and precisely two even-valence second-nearest neighbors. This can be proven by considering the local empire of the 6vertex in Fig. 4. This region is large enough to cover the entire tiling, allowing for overlaps [81]. In Fig. 6, the local empire of the 6-vertex is shown; the solid thick black lines connect second-nearest-neighbor even-valence vertices (note that we include an extra twig on the 6-vertex; this is explained shortly). The thick dashed black lines represent potential completions of the loop, which could become solid depending on how the incomplete boundary vertices are finished (i.e., the precise way in which each vertex appears in the tiling). The 6-vertex has zero even-valence neighbors, and two 4-vertices are the only even-valence second-nearest neighbors. Each of these 4-vertices has no even-valence neighbors, and, aside from the 6-vertex, may have either a 4-vertex or 6-vertex as another second-nearest neighbor (by considering all possible completions of the boundary vertices). Elsewhere in the local empire, an arc of three second-nearest-neighbor 4-vertices appears. Again considering all possible completions of boundary vertices, the only possible continuations are to a $-4-6-4-4-$ 4-6-4- configuration, where only second-nearestneighbor even-valence vertices are listed, or a $4^{5}$-loop. This completes the proof that even-valence vertices have zero even-valence vertices as neighbors and precisely two even-valence vertices as second-nearest neighbors.

\section{Absence of monomers on monomer membranes in maximum matchings}

A key result in the main text is that monomers never appear on monomer membranes. In the main text, we prove, using a graphical argument that considers implication networks, that 6-vertices cannot host monomers. We now use a similar approach to show that monomers cannot appear on 4 -vertices, or on $5_{C}$-vertices appearing between 4-vertices and 6-vertices, in a maximum matching. Collectively, these vertices comprise closed loops which monomers cannot cross, which we term monomer membranes in the main text. Note that monomers can appear on $5_{C}$-vertices appearing between two 4-vertices but only in the exceptional case of the $4^{5}$-loop (see Sec. III).

In Fig. 19, we prove that no monomer can appear on a 4 -vertex in a maximum matching. The local empire of the 4-vertex is too small to construct a direct analog to the argument presented for the 6-vertex. However, 4-vertices have either 4-vertices or 6-vertices as second-nearest neighbors (with $5_{C}$-vertices connecting them). Figures 19(a)19(d) assume a -4 - 6- configuration, where the boxed symbol is the vertex under consideration and the $5_{C}$ connecting vertices are not listed. Figures 19(e)-19(h) assume a $-4-4-4-$ configuration. Place a monomer (blue) on the 4-vertex. The circled second-nearest neighbor can host dimers in one of three symmetry-inequivalent positions [Figs. 19(a)-19(c)], or it can host a monomer [Fig. 19(d)]. In all cases, it can be seen that at least two further monomers are implied which connect via augmenting paths to the monomer on the 4-vertex (a contradiction). In Figs. 19(e)-19(h), the same argument applies in the other configuration. Therefore, a monomer cannot appear on a 4-vertex in a maximum dimer matching.

In Fig. 20, we prove that no monomer can be based on a $5_{C}$-vertex appearing between a 4 -vertex and a 6 -vertex in a maximum matching. Place a monomer (blue) on the $5_{C}$-vertex. The circled vertex must host a dimer if an augmenting path is to be avoided. The two options are 

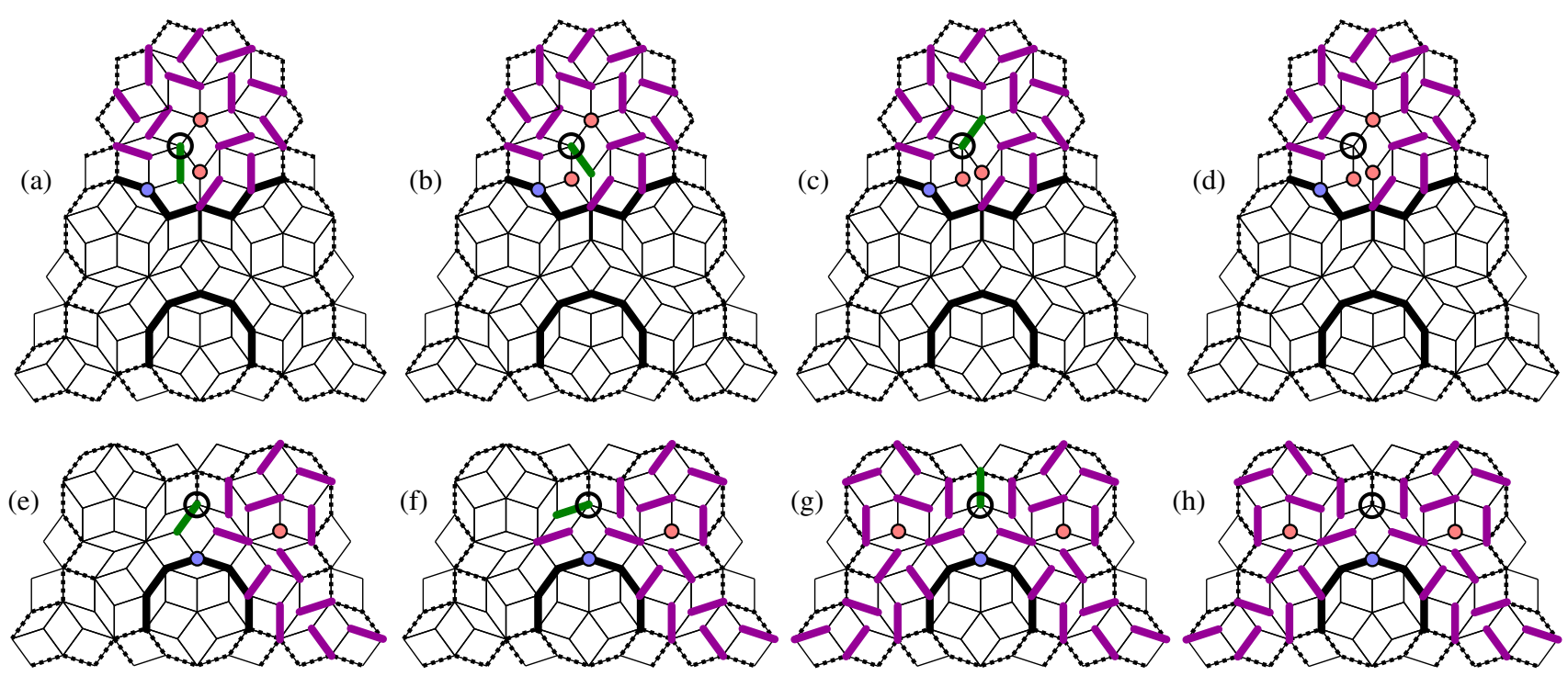

FIG. 19. Proof no monomer can be based on a 4-vertex in a maximum matching. See accompanying text in Appendix A 2.

shown in Figs. 20(a) and 20(b), and in both cases, the second monomer is shown to be unavoidable.

Finally, note that when a $5_{C}$-vertex appears as part of an impermeable monomer membrane (i.e., any membrane larger than $4^{5}$ ), the set of edges which can be covered by dimers is restricted. The easiest way to state the restriction is that the edges which cannot be covered are those which are either already implied by the uncoverable edges of 4or -6-vertices, and the edge lying along the mirror plane of the $5_{C}$-vertex whenever deletion of this edge will lead to a disconnected graph. In the $-4-55_{C}-6-$ configuration, this result is proven as part of the proofs just given for the 4- and 6-vertices (the relevant uncoverable legs of the $5_{C^{-}}$ vertex are the uncoverable legs of the 4- or 6-vertex). In the $4^{5}$ configuration, the result does not hold, and all legs of the $5_{C}$-vertex are potentially coverable. The only remaining case is the $-4-6-4-55_{C}-4-4-6-4-$ configuration (where only the relevant $5_{C}$-vertex is listed). Two of the uncoverable legs are again proven uncoverable by the

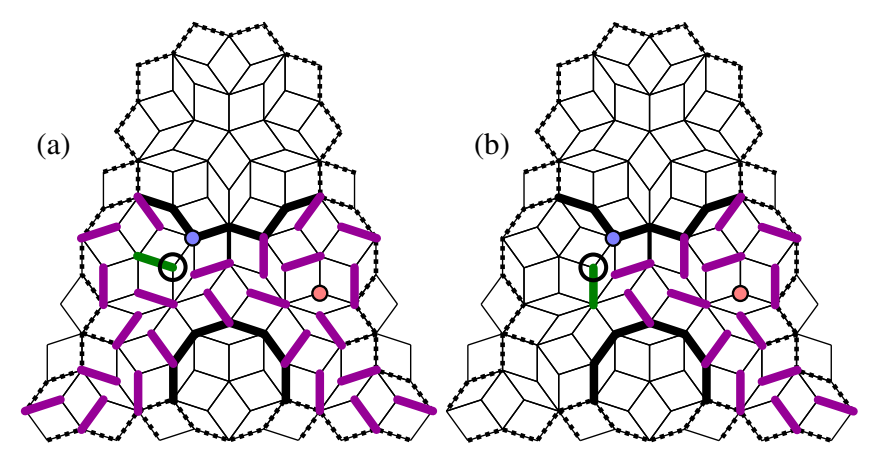

FIG. 20. Proof no monomer can be based on a $5_{C}$-vertex appearing between a 4-vertex and a 6-vertex in a maximum matching. See accompanying text in Appendix A 2. 4-vertex proof. The remaining case also follows directly from Fig. 22(c).

\section{Restrictions on dimer placement}

We now prove that in any maximum matching, an evenvalence vertex must always touch a dimer, which must always cover one of the edges indicated in Fig. 6(b). The first part of the statement follows from the absence of monomers on even-valence vertices in maximum matchings. The second can almost be seen directly from the proof presented in Fig. 6(a): The monomer placed on the 6-vertex could equally well be a dimer protruding downwards [any of the three options indicated as disallowed in Fig. 6(b)]. However, there would be no immediate problem with a monomer residing next to such a dimer. To complete the proof, we need only to show that a monomer of the opposite bipartite charge always resides on the other side of the thick black line.

In Fig. 21, we prove that the dimer which must connect to the 6-vertex must appear on one of the three legs indicated in Fig. 6(b). The proof takes the following form. Based on the results of Figs. 6 and 19, no monomer can exist on 4-vertices or 6-vertices. The proofs in those figures also demonstrate that if the monomer is substituted with a dimer extending into the region on the opposite side of the thick black line, augmenting paths can exist which include this dimer. After augmentation, the dimer will be returned to one of the edges specified in Fig. 6(b). Rephrasing in terms of minimal monomer moves, exactly one monomer will be able to cross the black line. This cannot occur in a maximum matching if there is a monomer of opposite bipartite charge waiting on the other side of the thick black line, as the two monomers will then be able to annihilate, and should not have been present in a maximum matching. In Figs. 21(a) and 21(b), above the thick black line we 

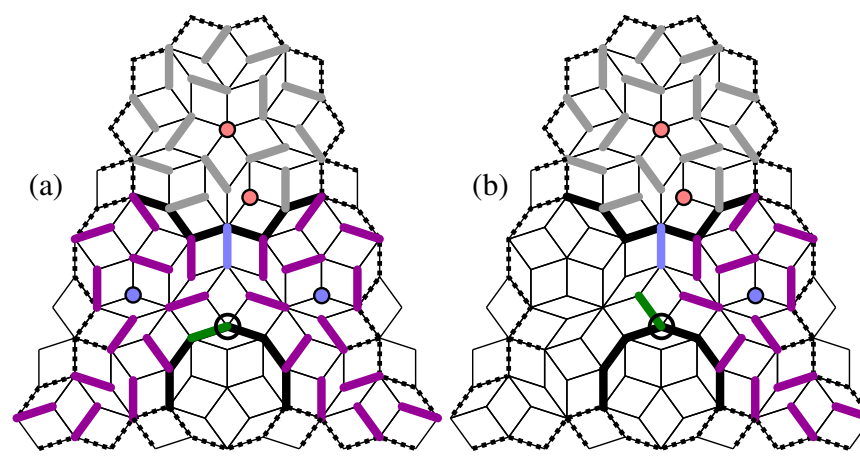

(c)

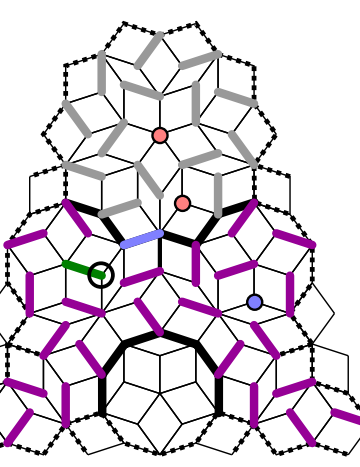

(d)

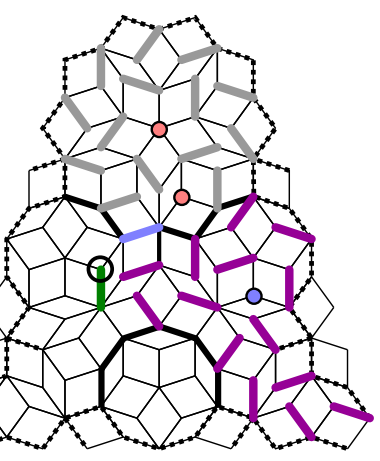

FIG. 21. Proof that, of the edges emanating from a 6-vertex, only the edges indicated in Fig. 6(b) may host dimers. See accompanying text in Appendix A 2.

reproduce one of the maximum matchings from Fig. 6(a), with the dimers recolored to gray to indicate that the specific configuration is unimportant to the argument (any case will work). We assume the 6-vertex does not host a monomer but instead hosts the forbidden dimer indicated in blue. By considering the possible edges of the circled 4-vertex directly below, of which there are two symmetryinequivalent choices, we show the implied dimers in each case, and in each case a blue monomer is implied with the opposite charge to the monomers in the region above the line. As the blue dimer connects the regions, there exists an augmenting path (i.e., exactly one monomer can cross the wall to annihilate), and the matching is not maximum. Note that the choice of the circled 4-vertex is simply out of convenience, and other vertices could have been considered. In Fig. 21(b), we construct a similar proof for the remaining symmetry-inequivalent edge of the 6-vertex, again covered by a dimer indicated in blue, and this time the circled 3 -vertex is convenient to consider.
In Fig. 22, we prove that the dimer which must connect to the 4-vertex must appear on one of the two legs indicated in Fig. 6(b). The case of the 4-vertex is complicated by the relatively smaller local empire of the 4-vertex. Three cases need to be considered, recalling that any even-valence vertex has two even-valence vertices as second-nearest neighbors. First, the 4-vertex may have a 6-vertex as a second-nearest neighbor, as in Figs. 22(a) and 22(b). In this case, we may again consider the local empire of the 6-vertex. We place the blue dimer on each of the edges in question, and we consider a convenient nearby vertex. In this case, the nearby $5_{A}$-vertex works well (shown). The five edges connected to this vertex must be considered, of which only one choice is shown in Figs. 22(a) and 22(b). The other cases can be checked quickly and yield the familiar result, that the forbidden placement of the blue dimer allows a single monomer to cross the black line and annihilate with an oppositely charged monomer, so this configuration will not appear in a maximum matching.
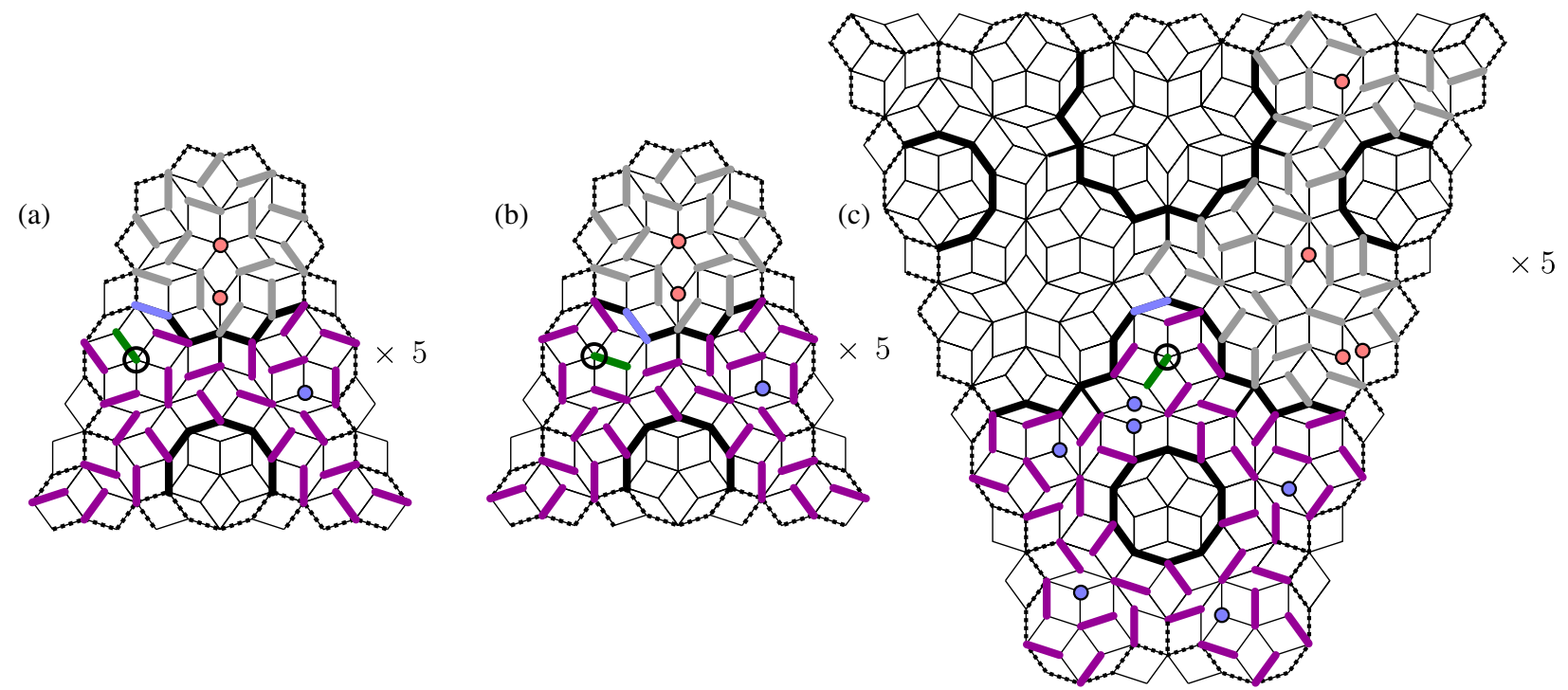

FIG. 22. Proof that, of the edges emanating from a 4-vertex, only the edges indicated in Fig. 6(b) may host dimers. See accompanying text in Appendix A 2 and Fig. 21. 
The second option is that the 4-vertex has 4-vertices on either side but that the vertex after that is a 6 -vertex. The local empire of this configuration is shown in Fig. 22(c); in fact, it implies the chain $-4-6-4-4-4-6-4-$ as shown. There is only one symmetry-inequivalent edge to consider; choose again the circled neighboring $5_{A}$-vertex, and any of the five dimer placements implies blue monomers to annihilate with the red monomers in the other region. The final option is that the 4 -vertex appears in a $4^{5}$ ring [also present elsewhere in Fig. 22(c)]. In this case, the proof cannot be constructed, as this is the only case in which the otherwise-forbidden edges of the 4-vertex can be covered by a dimer. Only one of the five such edges may be covered by a dimer, and this is only if there is no monomer within the ring.

Therefore, if present, the forbidden dimer will permit an augmenting path (which crosses the line). In a maximum matching, this situation can never arise, since by definition, augmenting paths cannot exist. Rephrasing in terms of minimal monomer moves, the forbidden dimer would allow the passage of precisely one monomer over the line, which could then annihilate with a monomer of opposite charge.

\section{Proof of the impermeability of monomer membranes by monomers}

We now show that monomer membranes provide impenetrable barriers to monomer motion. To see this, observe that each closed loop bounds two regions. On the side of the loop into which the even-valence vertices point [with their directions indicated in Fig. 6(b)], a dimer will protrude from each even-valence vertex. In principle, this dimer could provide one end of an alternating path, the other end of which terminates on a monomer. However, the monomers in this region are all of the opposite bipartite charge to the even-valence vertices comprising the membrane, so this situation cannot occur. On the side of the loop away from which the even-valence vertices point, there exist monomers of the same bipartite charge as the even-valence vertices-but no dimers connect from the even-valence vertices into this region, and, since alternating paths start on monomers and end on the dimer connected to a vertex, no alternating path can reach these vertices. The situation is reversed for the $5_{C}$-vertices constituting the remaining members of the loops, which have opposite bipartite charge to the even-valence vertices.

\section{APPENDIX B: PROOF THAT THE DIMER INFLATION ALGORITHM GENERATES MAXIMUM MATCHINGS}

In this Appendix, we prove the following statements made in Sec. IV B:

(i) Any path connecting any two 7-vertices is of even (odd) length if it crosses impermeable monomer membranes an even (odd) number of times. (ii) Any path connecting any two $5_{A, B}$-vertices, where the $5_{A, B}$-vertices have no 7 -vertices as secondnearest neighbors, is of even (odd) length if it crosses impermeable monomer membranes an even (odd) number of times.

(iii) Any path connecting any 7-vertex to any $5_{A, B^{-}}$ vertices, where the $5_{A, B}$-vertices have no 7 -vertices as second-nearest neighbors, is of odd (even) length if it crosses impermeable monomer membranes an even (odd) number of times.

Figure 11 shows the local empire of the 6-vertex, sufficient to cover the Penrose tiling. Colored disks indicate vertices of certain valences which are either definite (solid circles) or potential, depending on the surrounding patches (dashed circles). Red vertices are 7-vertices; blue vertices are $5_{A}$-vertices; pink vertices are $5_{B}$-vertices. The thick solid line indicating the monomer membrane boundary passing through the 6-vertex disconnects vertices $a-g$ plus the internal red and blue vertices, from vertices $h-k$ and the internal pink vertex, in all possible continuations of the monomer membrane. Note that all internal vertices obey the specified rules, and so, when considering a boundary vertex, its relationship needs only to be shown to be correct to any one internal vertex.

(i) Red vertices $c, e$, and $g$ are connected by evenlength paths to the interior red vertices.

(ii) All these vertices are connected by odd-length paths to red vertices $h$ and $j$, which must be separated by a monomer membrane (if $h$ is a 7-vertex it implies $g$ is not, as the potential monomer membrane is resolved to pass downwards, implying $g$ is a $5_{C}$-vertex).

(iii) Red vertex $b$ is connected by odd-length paths to the interior red vertices. However, if $b$ is a 7-vertex, $c$ (and its mirror equivalent) is forced to be a 6-vertex, in which case, $b$ is separated from the interior red vertices by an impermeable monomer membrane.

(iv) Blue vertex $a$ is connected to the interior blue vertices by odd-length paths. If $b$ is a 4-vertex then $a$ is a $5_{A}$-vertex with no 7 -vertices as second-nearest neighbors, so $a$ receives a monomer of the same bipartite charge, and since the interior blue vertices have at least one 7-vertex as a second-nearest neighbor, they receive monomers of opposite bipartite charge to themselves, i.e., the same as the charge of $a$. This is correct. If $b$ is not a 4-vertex, it must be a 7-vertex. The monomer associated with $a$ is now of the opposite bipartite charge to $a$, but if $b$ is not a 4 -vertex then $c$ must be a 6 -vertex to continue the monomer membrane, and so $a$ is separated by an impermeable monomer membrane from the interior blue vertices. This too is correct.

(v) Blue vertex $d$ is connected by even-length paths to the internal blue vertices.

(vi) Pink vertex $f$ is connected by odd-length paths to the internal red vertices. 
(vii) Red vertex $h$ is connected by odd-length paths to the internal pink vertex.

(viii) Blue vertex $i$ is connected by odd-length paths to the internal pink vertex. If $i$ is a $5_{A}$-vertex then $h$ and $j$ must be 7-vertices, and so the monomers associated with $i$ are of opposite bipartite charge to $i$. As the monomer associated with the pink vertex is of the same charge as the pink, this is correct. If $i$ is a 4 -vertex, $h$ must be a 6-vertex, and there is no issue with the presence of the internal pink vertex.

(ix) Similarly, if either $h$ or $j$ is a 7-vertex, this forces the $i$ to be a $5_{A}$-vertex, and the same cases hold. The same cases also hold for $k$.

This concludes the check of every possible boundary vertex of interest on the local empire of the 6-vertex (the unmarked boundary vertices either cannot be $5_{A^{-}}, 5_{B^{-}}$, or 7 -vertices, or the results are implied by the vertical mirror plane passing through the 6-vertex).

[1] D. S. Rokhsar and S. A. Kivelson, Superconductivity and the Quantum Hard-Core Dimer Gas, Phys. Rev. Lett. 61, 2376 (1988).

[2] S. A. Kivelson, D. S. Rokhsar, and J. P. Sethna, Topology of the Resonating Valence-Bond State: Solitons and High$T_{c}$ Superconductivity, Phys. Rev. B 35, 8865 (1987).

[3] R. Moessner and S. L. Sondhi, Resonating Valence Bond Phase in the Triangular Lattice Quantum Dimer Model, Phys. Rev. Lett. 86, 1881 (2001).

[4] R. Moessner and K. S. Raman, Quantum Dimer Models (Lecture Notes, Trieste, 2007).

[5] P. Fazekas and P. W. Anderson, On the Ground State Properties of the Anisotropic Triangular Antiferromagnet, Philos. Mag. 30, 423 (1974).

[6] P. W. Anderson, The Resonating Valence Bond State in $\mathrm{La}_{2} \mathrm{CuO}_{4}$ and Superconductivity, Science 235, 1196 (1987).

[7] L. Savary and L. Balents, Quantum Spin Liquids: A Review, Rep. Prog. Phys. 80, 016502 (2017).

[8] C. Nayak, S. H. Simon, A. Stern, M. Freedman, and S. D. Sarma, Non-Abelian Anyons and Topological Quantum Computation, Rev. Mod. Phys. 80, 1083 (2008).

[9] R. Moessner, S. L. Sondhi, and E. Fradkin, Short-Ranged Resonating Valence Bond Physics, Quantum Dimer Models, and Ising Gauge Theories, Phys. Rev. B 65, 024504 (2001).

[10] R. Moessner and S. L. Sondhi, Three-Dimensional Resonating-Valence-Bond Liquids and Their Excitations, Phys. Rev. B 68, 184512 (2003).

[11] P. Kasteleyn, The Statistics of Dimers on a Lattice: I. The Number of Dimer Arrangements on a Quadratic Lattice, Physica 27, 1209 (1961).

[12] M. E. Fisher, Statistical Mechanics of Dimers on a Plane Lattice, Phys. Rev. 124, 1664 (1961).

[13] H. N. V. Temperley and M. E. Fisher, Dimer Problem in Statistical Mechanics-An Exact Result, Philos. Mag. 6, 1061 (1961).
[14] P. Kasteleyn, Graph Theory and Crystal Physics, in Graph Theory and Theoretical Physics, edited by F. Harary (Academic Press, London, 1967), pp. 43-110.

[15] C. Hurst and H. Green, New Solution to the Ising Problem for a Rectangular Lattice, J. Chem. Phys. 33, 1059 (1960).

[16] D. A. Huse, W. Krauth, R. Moessner, and S. L. Sondhi, Coulomb and Liquid Dimer Models in Three Dimensions, Phys. Rev. Lett. 91, 167004 (2003).

[17] W. Krauth and R. Moessner, Pocket Monte Carlo Algorithm for Classical Doped Dimer Models, Phys. Rev. B 67, 064503 (2003).

[18] C. Richard, M. Höffe, J. Hermisson, and M. Baake, Random Tilings: Concepts and Examples, J. Phys. A 31, 6385 (1998).

[19] R. Kenyon, J. G. Propp, and D. B. Wilson, Trees and Matchings, Electron. J. Comb. 7, R25 (2000).

[20] R. Kenyon, The Laplacian and Dirac Operators on Critical Planar Graphs, Inventiones Mathematicae 150, 409 (2002).

[21] R. Kenyon and A. Okounkov, Planar Dimers and Harnack Curves, Duke Math. J. 131, 499 (2006).

[22] R. Kenyon, A. Okounkov, and S. Sheffield, Dimers and Amoebae, arXiv:math-ph/0311005.

[23] R. Dijkgraaf, D. Orlando, and S. Reffert, Dimer Models, Free Fermions and Super Quantum Mechanics, arXiv:0705.1645.

[24] P. L. Krapivsky, S. Redner, and E. Ben-Naim, A Kinetic View of Statistical Physics (Cambridge University Press, Cambridge, England, 2010).

[25] P. Erdős and A. Rényi, On Random Graphs I., Publ. Math. (Debrecen) 6, 290 (1959).

[26] M. E. J. Newman, S. H. Strogatz, and D. J. Watts, Random Graphs with Arbitrary Degree Distributions and Their Applications, Phys. Rev. E 64, 026118 (2001).

[27] D. Alberici, P. Contucci, and E. Mingione, A Mean-Field Monomer-Dimer Model with Randomness: Exact Solution and Rigorous Results, J. Stat. Phys. 160, 1721 (2015).

[28] D. Shechtman, I. Blech, D. Gratias, and J. W. Cahn, Metallic Phase with Long-Range Orientational Order and No Translational Symmetry, Phys. Rev. Lett. 53, 1951 (1984).

[29] R. Penrose, The Role of Aesthetics in Pure and Applied Mathematical Research, Bull. Inst. Math. Appl. 10, 266 (1974).

[30] M. Senechal, Quasicrystals and Geometry (Cambridge University Press, Cambridge, England, 1995).

[31] C. Janot, Quasicrystals: A Primer (Oxford University Press, New York, 1994).

[32] B. Grünbaum and G. C. Shephard, Tilings and Patterns (W. H. Freeman and Company, New York, 1986).

[33] We use the phrase Penrose-like tiling to indicate aperiodic tilings featuring diffraction patterns (Fourier transforms) with Bragg peaks and dense backgrounds which feature rotational symmetries forbidden by crystallographic restriction. We use the phrase quasilattice for their onedimensional equivalents.

[34] J. E. S. Socolar, T. C. Lubensky, and P. J. Steinhardt, Phonons, Phasons, and Dislocations in Quasicrystals, Phys. Rev. B 34, 3345 (1986).

[35] M. Kohmoto, B. Sutherland, and C. Tang, Critical Wave Functions and a Cantor-Set Spectrum of a 
One-Dimensional Quasicrystal Model, Phys. Rev. B 35, 1020 (1987).

[36] J. A. Ashraff, J.-M. Luck, and R. B. Stinchcombe, Dynamical Properties of Two-Dimensional Quasicrystals, Phys. Rev. B 41, 4314 (1990).

[37] Y.E. Kraus, Y. Lahini, Z. Ringel, M. Verbin, and O. Zilberberg, Topological States and Adiabatic Pumping in Quasicrystals, Phys. Rev. Lett. 109, 106402 (2012).

[38] K. A. Madsen, E. J. Bergholtz, and P. W. Brouwer, Topological Equivalence of Crystal and Quasicrystal Band Structures, Phys. Rev. B 88, 125118 (2013).

[39] F. Flicker and J. van Wezel, Quasiperiodicity and $2 D$ Topology in $1 D$ Charge-Ordered Materials, Europhys. Lett. 111, 37008 (2015).

[40] S. Iyer, V. Oganesyan, G. Refael, and D. A. Huse, ManyBody Localization in a Quasiperiodic System, Phys. Rev. B 87, 134202 (2013).

[41] M. Schreiber, S. S. Hodgman, P. Bordia, H. P. Lüschen, M. H. Fischer, R. Vosk, E. Altman, U. Schneider, and I. Bloch, Observation of Many-Body Localization of Interacting Fermions in a Quasirandom Optical Lattice, Science 349, 842 (2015).

[42] V. Khemani, D. N. Sheng, and D. A. Huse, Two Universality Classes for the Many-Body Localization Transition, Phys. Rev. Lett. 119, 075702 (2017).

[43] A. Jagannathan, Quasiperiodic Heisenberg Antiferromagnets in Two Dimensions, Eur. Phys. J. B 85, 68 (2012).

[44] S. Thiem and J. T. Chalker, Long-Range Magnetic Order in Models for Rare-Earth Quasicrystals, Phys. Rev. B 92, 224409 (2015).

[45] S. Thiem and J. T. Chalker, Magnetism in Rare-Earth Quasicrystals: RKKY Interactions and Ordering, Europhys. Lett. 110, 17002 (2015).

[46] E. C. Andrade, A. Jagannathan, E. Miranda, M. Vojta, and V. Dobrosavljević, Non-Fermi-Liquid Behavior in Metallic Quasicrystals with Local Magnetic Moments, Phys. Rev. Lett. 115, 036403 (2015).

[47] N. K. Sato, S. Matsukawa, K. Nobe, K. Imura, K. Deguchi, and T. Ishimasa, Quantum Critical Behavior in Magnetic Quasicrystals and Approximant Crystals, J. Phys. Conf. Ser. 868, 012005 (2017).

[48] K. Kamiya, T. Takeuchi, N. Kabeya, N. Wada, T. Ishimasa, A. Ochiai, K. Deguchi, K. Imura, and N. K. Sato, Discovery of Superconductivity in Quasicrystal, Nat. Commun. 9, 154 (2018).

[49] J. Kepler, Harmonices Mundi (J. Planck, Linz, Austria, 1619).

[50] L. S. Levitov, Why Only Quadratic Irrationalities are Observed in Quasi-Crystals?, Europhys. Lett. 6, 517 (1988).

[51] N. W. Ashcroft and N. D. Mermin, Solid State Physics (Harcourt College Publishers, New York, 1976).

[52] N. de Bruijn, Algebraic Theory of Penrose's Non-Periodic Tilings of the Plane. I, Indagat. Math 84, 39 (1981).

[53] C. L. Henley, Sphere Packings and Local Environments in Penrose Tilings, Phys. Rev. B 34, 797 (1986).

[54] M. Gardner, Penrose Tiles to Trapdoor Ciphers... and the Return of Dr. Matrix (Mathematical Association of America, Oberlin, OH, 1989).
[55] L. Boyle and P. Steinhardt, Self-Similar One-Dimensional Quasilattices, arXiv:1608.08220.

[56] L. Boyle and P. Steinhardt, Coxeter Pairs, Ammann Patterns and Penrose-like Tilings, arXiv:1608.08215.

[57] E. Bombieri and J. Taylor, Which Distributions of Matter Diffract? An Initial Investigation, J. Phys. (Paris), Colloq. 47, C3 (1986).

[58] L. Zaporski and F. Flicker, Superconvergence of Topological Entropy in the Symbolic Dynamics of Substitution Sequences, SciPost Phys. 7, 018 (2019).

[59] L. Effinger-Dean, The Empire Problem in Penrose Tilings, B. A. thesis, Williams College, 2006.

[60] A. Gibbons, Algorithmic Graph Theory (Cambridge University Press, Cambridge, England, 1985).

[61] R. J. Baxter, Exactly Solved Models in Statistical Mechanics (Academic Press, London, 1982).

[62] J. E. Hopcroft and R. M. Karp, An $n^{5 / 2}$ Algorithm for Maximum Matchings in Bipartite Graphs, SIAM J. Comput. 2, 225 (1973).

[63] Note that paths are distinct from walks: Paths are walks that cannot self-intersect.

[64] P. A. M. Dirac, Quantised Singularities in the Electromagnetic Field, Proc. R. Soc. A 133, 60 (1931).

[65] C. Mercat, Discrete Riemann Surfaces and the Ising Model, Commun. Math. Phys. 218, 177 (2001).

[66] B. de Tilière, Quadri-Tilings of the Plane, Probab. Theory Relat. Fields 137, 487 (2007).

[67] V. E. Korepin, Completely Integrable Models in Quasicrystals, Commun. Math. Phys. 110, 157 (1987).

[68] E. Y. Vedmedenko, H. P. Oepen, and J. Kirschner, Decagonal Quasiferromagnetic Microstructure on the Penrose Tiling, Phys. Rev. Lett. 90, 137203 (2003).

[69] E. Y. Vedmedenko, U. Grimm, and R. Wiesendanger, Noncollinear Magnetic Order in Quasicrystals, Phys. Rev. Lett. 93, 076407 (2004).

[70] H. Au-Yang and J. Perk, Q-dependent Susceptibilities in Z-invariant Pentagrid Ising Models, J. Stat. Phys. 127, 221 (2007).

[71] A. Koga and H. Tsunetsugu, Antiferromagnetic Order in the Hubbard Model on the Penrose Lattice, Phys. Rev. B 96, 214402 (2017).

[72] M. Arai, T. Tokihiro, T. Fujiwara, and M. Kohmoto, Strictly Localized States on a Two-Dimensional Penrose Lattice, Phys. Rev. B 38, 1621 (1988).

[73] M. Kohmoto and B. Sutherland, Electronic States on a Penrose Lattice, Phys. Rev. Lett. 56, 2740 (1986).

[74] B. Sutherland, Localization of Electronic Wave Functions due to Local Topology, Phys. Rev. B 34, 5208 (1986).

[75] H. C. Longuet-Higgins, Some Studies in Molecular Orbital Theory I. Resonance Structures and Molecular Orbitals in Unsaturated Hydrocarbons, J. Chem. Phys. 18, 265 (1950).

[76] We thank K. Damle for bringing these facts to our attention.

[77] D. H. Dunlap, H.-L. Wu, and P. W. Phillips, Absence of Localization in a Random-Dimer Model, Phys. Rev. Lett. 65, 88 (1990).

[78] A. B. Kempe, On the Geographical Problem of the Four Colours, Am. J. Math. 2, 193 (1879). 
[79] A. Soifer, The Mathematical Coloring Book (SpringerVerlag, New York, 2009).

[80] Note that we use the term nearest or next-nearest neighbor, etc., in the sense of graph connectivity rather than actual spatial distance.

[81] P. Gummelt, Penrose Tilings as Coverings of Congruent Decagons, Geometriae Dedicata 62, 1 (1996).

[82] We note that PYTHON's NetworkX implementation of the Hopcroft-Karp algorithm never seems to place monomers on the branching points of implication networks, despite the fact that the monomers it does place connect via alternating paths to these points. See, e.g., Fig. 7.

[83] The sequence of central vertices under repeated inflation is $5_{A}, 5_{B}, 5_{A}$ rotated through $2 \pi / 10,5_{B}$ rotated through $2 \pi / 10$, after which the sequence repeats. Note that an arbitrary vertex takes at most three inflations to reach the sequence.

[84] Note that these inflation rules also govern the itinerary of the period-doubling cascade into chaos in unimodal maps (see Ref. [58] and references therein).

[85] M. V. Jarić, Diffraction from Quasicrystals: Geometric Structure Factor, Phys. Rev. B 34, 4685 (1986).

[86] J. Peyriere, Frequency of Patterns in Certain Graphs and in Penrose Tilings, J. Phys. (Paris), Colloq. 47, C3 (1986).

[87] R. Moessner and J. T. Chalker, Low-Temperature Properties of Classical Geometrically Frustrated Antiferromagnets, Phys. Rev. B 58, 12049 (1998).

[88] L. Boltzmann, Vorlesungen :uber Gastheorie (J. A. Barth, Leipzig, 1898).

[89] T. Uno, in Proceedings of the 8th International Symposium on Algorithms and Computation, ISAAC '97 (SpringerVerlag, London, 1997), pp. 92-101.

[90] M. Baake and U. Grimm, Aperiodic Order Volume 1: A Mathematical Invitation (Cambridge University Press, Cambridge, England, 2013).

[91] This design was developed during discussions with N. G. Jones.

[92] J. Lloyd, S. H. Simon, S. A. Parameswaran, and F. Flicker, Perfect Dimer Matchings of Ammann-Beenker Tilings (to be published).

[93] J. E. S. Socolar, Simple Octagonal and Dodecagonal Quasicrystals, Phys. Rev. B 39, 10519 (1989).

[94] L. Lovász and M. D. Plummer, Matching Theory (NorthHolland, Amsterdam, 1986).

[95] R. McGrath, J. Ledieu, E. Cox, S. Haq, R. Diehl, C. Jenks, I. Fisher, A. Ross, and T. Lograsso, Quasicrystal Surfaces: Potential as Templates for Molecular Adsorption, J. Alloys Compd. 342, 432 (2002).

[96] R. A. Trasca, N. Ferralis, R. D. Diehl, and M. W. Cole, The Adsorption of Xe and Ar on Quasicrystalline Al-Ni-Co, J. Phys. Condens. Matter 16, S2911 (2004).

[97] R. McGrath, Quasicrystal Surfaces: Structure, Adsorption and Epitaxy, Adv. Mater. Res. 545, 43 (2012).

[98] C. Castelnovo, R. Moessner, and S. L. Sondhi, Magnetic Monopoles in Spin Ice, Nature (London) 451, 42 (2008).

[99] D. J. P. Morris, D. A. Tennant, S. A. Grigera, B. Klemke, C. Castelnovo, R. Moessner, C. Czternasty, M. Meissner, K. C. Rule, J.-U. Hoffmann, K. Kiefer, S. Gerischer, D. Slobinsky, and R. S. Perry, Dirac Strings and Magnetic Monopoles in the Spin Ice $\mathrm{Dy}_{2} \mathrm{Ti}_{2} \mathrm{O}_{7}$, Science 326, 411 (2009).
[100] S. T. Bramwell, S. R. Giblin, S. Calder, R. Aldus, D. Prabhakaran, and T. Fennell, Measurement of the Charge and Current of Magnetic Monopoles in Spin Ice, Nature (London) 461, 956 (2009).

[101] Alternatively, we can view them as forming a facecentered-cubic lattice with a four-site basis.

[102] M. J. P. Gingras, Observing Monopoles in a Magnetic Analog of Ice, Science 326, 375 (2009).

[103] M. J. P. Gingras and P. A. McClarty, Quantum Spin Ice: A Search for Gapless Quantum Spin Liquids in Pyrochlore Magnets, Rep. Prog. Phys. 77, 056501 (2014).

[104] A. Polyakov, Quark Confinement and Topology of Gauge Theories, Nucl. Phys. B120, 429 (1977).

[105] E. Fradkin, D. A. Huse, R. Moessner, V. Oganesyan, and S. L. Sondhi, Bipartite Rokhsar-Kivelson Points and Cantor Deconfinement, Phys. Rev. B 69, 224415 (2004).

[106] A. Vishwanath, L. Balents, and T. Senthil, Quantum Criticality and Deconfinement in Phase Transitions between Valence Bond Solids, Phys. Rev. B 69, 224416 (2004).

[107] M. Hermele, T. Senthil, M. P. A. Fisher, P. A. Lee, N. Nagaosa, and X.-G. Wen, Stability of U(1) Spin Liquids in Two Dimensions, Phys. Rev. B 70, 214437 (2004).

[108] S. Ryu, O. I. Motrunich, J. Alicea, and M. P. A. Fisher, Algebraic Vortex Liquid Theory of a Quantum Antiferromagnet on the Kagome Lattice, Phys. Rev. B 75, 184406 (2007).

[109] C. Chamon, Quantum Glassiness in Strongly Correlated Clean Systems: An Example of Topological Overprotection, Phys. Rev. Lett. 94, 040402 (2005).

[110] S. Bravyi and B. Terhal, A No-Go Theorem for a TwoDimensional Self-Correcting Quantum Memory Based on Stabilizer Codes, New J. Phys. 11, 043029 (2009).

[111] J. Haah, Local Stabilizer Codes in Three Dimensions without String Logical Operators, Phys. Rev. A 83, 042330 (2011).

[112] R. M. Nandkishore and M. Hermele, Fractons, Annu. Rev. Condens. Matter Phys. 10, 295 (2019).

[113] A. Prem, J. Haah, and R. Nandkishore, Glassy Quantum Dynamics in Translation Invariant Fracton Models, Phys. Rev. B 95, 155133 (2017).

[114] M. E. J. Newman and C. Moore, Glassy Dynamics and Aging in an Exactly Solvable Spin Model, Phys. Rev. E 60, 5068 (1999).

[115] H. S. M. Coxeter, The Complete Enumeration of Finite Groups of the Form $R_{i}^{2}=\left(R_{i} R_{j}\right)^{k_{i j}}=1$, J. Math. Soc. s1-10, 21 (1935).

[116] F. Flicker, Time Quasilattices in Dissipative Dynamical Systems, SciPost Phys. 5, 001 (2018).

[117] T. Sibley and S. Wagon, Rhombic Penrose Tilings Can Be 3-Colored, Am. Math. Mon. 107, 251 (2000).

[118] R. Kenyon, Conformal Invariance of Domino Tiling, arXiv:math-ph/9910002.

[119] E. Ardonne, P. Fendley, and E. Fradkin, Topological Order and Conformal Quantum Critical Points, Ann. Phys. (Amsterdam) 310, 493 (2004).

[120] L. Boyle, M. Dickens, and F. Flicker, Conformal Quasicrystals and Holography, arXiv:1805.02665. 